\title{
Triple point shear-layers in gaseous detonation waves
}

\author{
L. Massa, ${ }^{*}$ J.M. Austin,${ }^{\dagger}$ T.L. Jackson,${ }^{\ddagger}$ \\ University of Illinois at Urbana-Champaign, Urbana IL 61801 \\ luca1@csar.uiuc.edu,jmaustin@uiuc.edu,tlj@csar.uiuc.edu
}

\begin{abstract}
Recent experiments have shown intriguing regions of intense luminescence or "hot spots" in the vicinity of triple point shear-layers in propagating gaseous detonation waves. Localized explosions have also been observed to develop in these fronts. These features were observed in higher effective activation energy mixtures, but not in lower effective activation energy mixtures. We investigate the possibility that the increased lead shock oscillation through a cell cycle in higher activation energy mixtures may result in a significantly increased disparity in the induction time on either side on the triple point shear-layer, increasing the probability that shear-layer instability may develop between reacted and unreacted gas streams. We carry out two-dimensional simulations with detailed chemical kinetics to examine the nature of the triple point shear-layer in three mixtures with different effective activation energy. In the low activation energy mixture, large scale vortical structures are observed to occur downstream of the ignition distance; these structures do not have a noticeable effect on the reaction. In higher effective activation energy mixtures, a transverse ignition front develops near the interface between the two gas streams and results in a rapidly propagating reaction front. The transverse ignition front develops due to molecular diffusion across the shear-layer between hot and cold gas streams.
\end{abstract}

\section{Introduction}

A detonation front propagating through a premixed combustible gas can be modeled as a one-dimensional, steady wave in which the dominant mechanism is shock-induced chemical-thermal branching. However, the reaction rate behind the shock is extremely sensitive to perturbations in the post-shock temperature and as a result gaseous detonation waves are always unstable. Past work ${ }^{1-6}$ has revealed an extremely complex structure: the front is unstable in three-dimensions and also highly unsteady. The strength of the lead shock oscillates periodically in time and in space and detonations exhibit systems of counter-propagating transverse shock waves, Fig. 1. Triple points are formed at the intersection of the lead shock and transverse wave system. The triple-point shear-layer separates premixed gas streams that have passed through portions of the lead shock with different strength.

Regions of intense chemiluminescence, for example Fig. 2 (a), and localized explosions have recently been experimentally observed in detonation fronts propagating in highly unstable mixtures with high effective activation energy. ${ }^{7,9}$ From corresponding schlieren images, it appears the "hotspots" occur in the vicinity of the triple point shear-layers in the latter portion of the detonation cell, prior to the triple point collision. ${ }^{7}$ Such local hotspots were not observed in modest effective activation energy mixtures.

The magnitude of the lead shock oscillation through the cell cycle has been found to increase with increasing activation energy ${ }^{8,10}$ while the transverse wave strength is calculated to remain relatively constant..$^{7,11,12}$ In high activation energy mixtures, a significant difference in the lead shock strength might therefore be expected at the triple point, resulting in a considerable disparity in the induction time across the shear-layer.

*Research Scientist. Center for Simulation of Advanced Rockets. Member AIAA.

$\dagger$ Assistant Professor,Department of Aerospace Engineering. Member AIAA.

¥Senior Research Scientist. Center for Simulation of Advanced Rockets. Senior Member AIAA. 


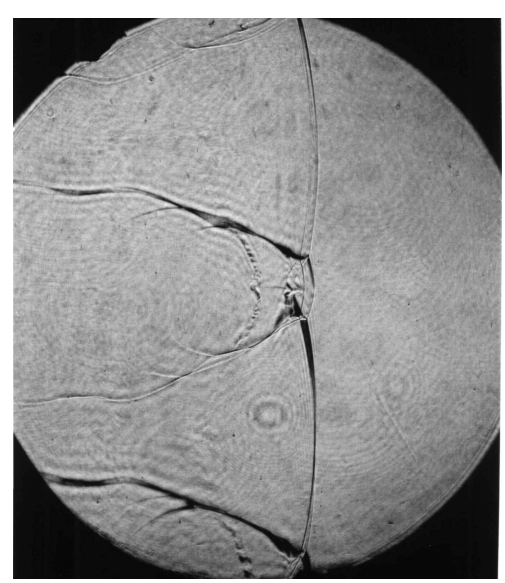

(a)

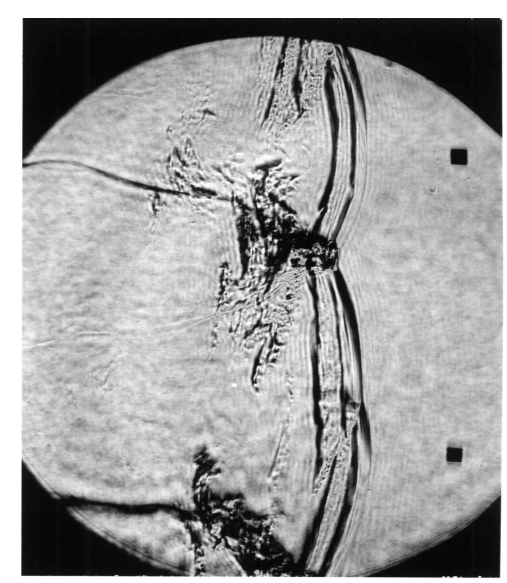

(b)

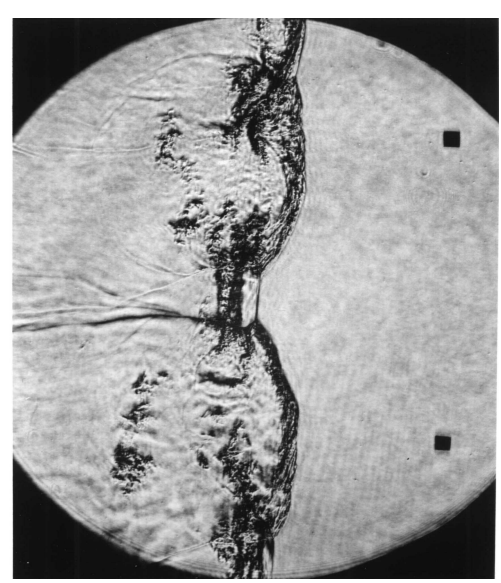

(c)

Figure 1: Schlieren images of detonation front propagating in the three mixtures considered in this study a) $2 \mathrm{H}_{2}-\mathrm{O}_{2}-17 \mathrm{Ar}$, b) $2 \mathrm{H}_{2}-\mathrm{O}_{2}-5.6 \mathrm{~N}_{2}$ c) $\mathrm{C}_{3} \mathrm{H}_{8}-5 \mathrm{O}_{2}-9 \mathrm{~N}_{2}$, all at initial pressure of $20 \mathrm{kPa}$. Detonations propagated left to right in a $152 \times 18 \mathrm{~mm}$ cross-section channel. ${ }^{7,8}$

Any local decoupling of the detonation front near the end of the cell cycle increases the induction time difference even more significantly. ${ }^{6,8,13}$ Thus in high activation energy mixtures, there is increased probability of shear-layer instabilities occurring between gas streams of different degree of reaction. Recent experiments have observed Kelvin-Helmholtz type instability between gas streams with different reactivity in detonation waves ${ }^{6,7}$ Fig. 2 (a). It is possible that molecular mixing of hot products and cold reactants may lead to hot spot formation and local explosions, critically augmenting the fundamental shock-induced combustion mechanism in the detonation front.

Motivated by these experiments, we examine the nature of the triple-point shear-layer in a gaseous detonation wave. The shear-layer consists of two premixed gas streams: one supersonic, unreacted stream that has passed through the incident and transverse waves, and one subsonic, reacted stream that has passed through the Mach stem, Fig 2 (c). Three mixtures are chosen to match experimental conditions. ${ }^{7}$ We find that the system has two ignition fronts, one perpendicular to the flow and associated with the thermal runaway, the other parallel to the stream and driven by molecular heating across the thermal layer. The physics underlying the second combustion is dominated by diffusion effects. The role of this second, diffusion controlled, front in the developing shear-layer system is of particular interest since diffusive processes are commonly thought to occur on time scales that are too long to be of relevance to detonations. This investigation focuses on the relation between reactivity of the system and the shear-layer instability. One of the main objectives is to elucidate the effect of the shear-layer instability in producing localized explosions or "hot spots" in the fresh gas stream. Since the induction distances are short, the linear stability characteristics of the shear-layer are considered for three mixtures with markedly different effective activation energies. The results of the linear analysis are elaborated on with two dimensional Navier-Stokes simulations with detailed chemistry and transport.

The possible role of diffusion in detonation waves has been previously addressed in several papers. Gamezo et al. ${ }^{14}$ speculate on the importance of diffusion in consuming unreacted gas pockets some distance downstream of the lead shock. Singh et al. ${ }^{15}$ showed that molecular and thermal transport effects are negligible in one-dimensional detonation waves. Arienti and Shepherd ${ }^{16}$ extended this work to evaluate the role of diffusion in triple point shear-layers in one-dimensional model problems. Time-resolved species evolution calculations using detailed chemical kinetics and simplified mixing models showed that the effect of diffusion depends on the mixture activation energy. Diffusion was negligible for modest activation energy mixtures, 


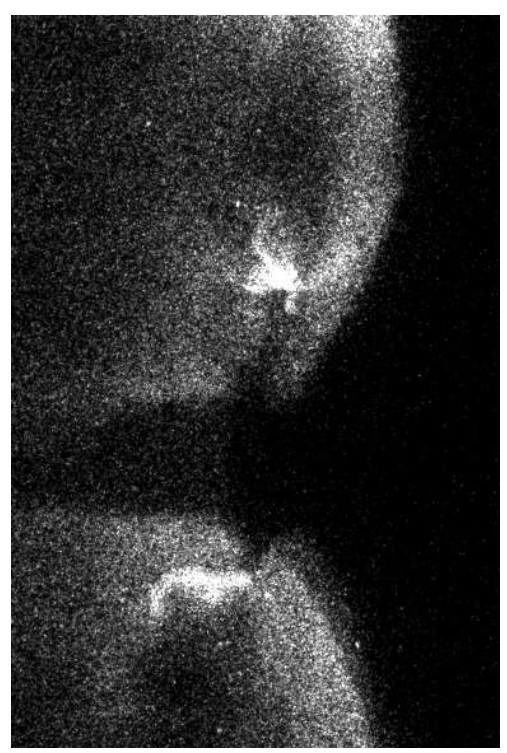

(a)

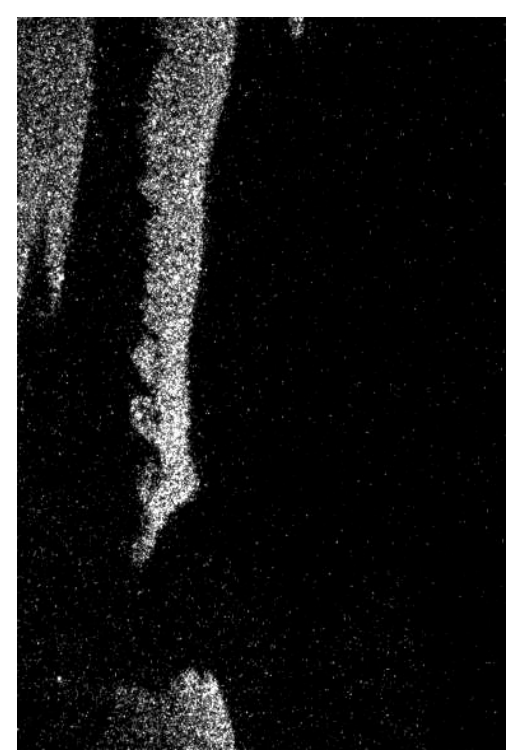

(b)

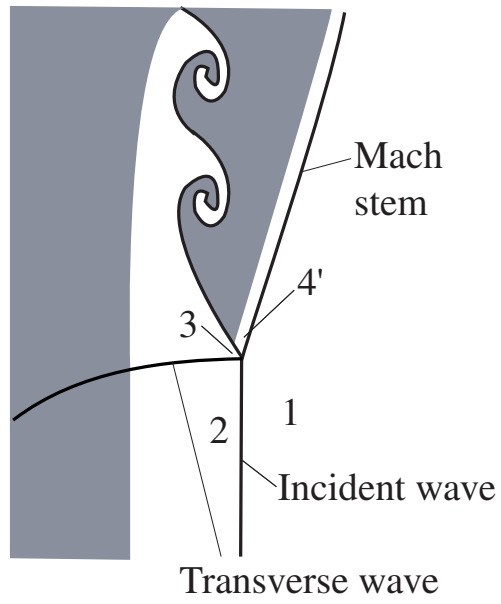

(c)

Figure 2: a) Regions of high fluorescence intensity or "hot spots" propagating gaseous detonation wave in a high effective activation energy mixture, $\mathrm{C}_{3} \mathrm{H}_{8}-5 \mathrm{O}_{2}-9 \mathrm{~N}_{2}, P_{1}=20 \mathrm{kPa} .^{7,8}$ Image height is $65 \mathrm{~mm}$. Simultaneous schlieren images indicate the hot spots occur in the vicinity of triple-point shear-layers near the end of the cell cycle but prior to the triple point collision. ${ }^{7}$ b) $\mathrm{OH}$ Planar laser-induced fluorescence image of a portion of a detonation front propagating in $2 \mathrm{H}_{2}-\mathrm{O}_{2}-7.7 \mathrm{~N}_{2}, P_{1}=20 \mathrm{kPa} \cdot{ }^{7}$ Kelvin-Helmholtz instabilities are evident between gases of different degree of $\mathrm{OH}$ number density. c) Schematic of a calculated triple point configuration with incident wave velocity $0.9 \mathrm{U}_{C J}$ and inflow angle $33^{\circ}$, corresponding to conditions near the end of the cell cycle. ${ }^{6}$ Grey shading indicates reacted gas. The triple point shear-layer separates gas which has passed through the incident and transverse waves (state 3), and gas which has passed through the Mach stem (state 4'). For the simulations, gas at state 4' is assumed to undergo a constant pressure reaction to state 4 .

but decreased the induction time in high activation energy mixtures. A temperature bulge followed by a laminar diffusion flame appeared in high activation energy mixtures when thermal and mass diffusion was included in the numerical calculation. We build on this existing work with two-dimensional simulations of the triple point shear-layer.

This article is organized as follows. First we present the initial conditions for the two gas streams in the three mixtures considered in this study. A linear stability analysis is carried out next, including an examination of the effects of finite chemistry, followed by a nonlinear analysis through numerical simulations of the two-dimensional Navier-Stokes equations with a detailed chemical kinetics mechanism.

\section{Initial conditions: Triple point calculation}

The initial conditions for the two streams on either side of the shear-layer are calculated using nonreactive shock polars. Shock polar calculations have been successfully used to analyze detonation triple points by several previous researchers. ${ }^{6,11,13,17}$ The flow is assumed to be steady in the triple point frame. Two parameters must be specified to close the calculation for conditions on either side of the contact surface. For all mixtures, the incoming velocity is taken to be $0.9 U_{C J}$, where $U_{C J}$ is the Chapman-Jouguet velocity. $0.9 U_{C J}$ is representative of the incident wave velocity near the end of the cell cycle. ${ }^{2,8,18}$ The incoming flow 
angle is taken to be $33^{\circ}$, a reasonable value based on soot foil track angles. More details on the triple point calculation procedure can be found in. ${ }^{7}$

Calculations show the triple point shear-layer separates a supersonic, relatively cold, gas stream and a subsonic, relatively hot, gas stream, states 3 and 4' respectively in Fig. 2 (c). In order to examine the role of mixing between unreacted and reacted gas streams as a potential mechanism for hot spot formation, we allow the subsonic stream to undergo constant pressure reaction from the chemically frozen post-shock state (state 4') to state 4 . This results in the initial conditions listed in Table 1 . Three mixture with different effective activation energy were considered. The mixtures were chosen to correspond to experimental conditions. ${ }^{7}$ In the paper, we will denote the mixture $2 \mathrm{H}_{2}-\mathrm{O}_{2}-17 \mathrm{Ar}$ as hydrogen-oxygen-argon system, the $2 \mathrm{H}_{2}-\mathrm{O}_{2}-5.6 \mathrm{~N}_{2}$ mixture as hydrogen-oxygen-nitrogen system, and the $\mathrm{C}_{3} \mathrm{H}_{8}-5 \mathrm{O}_{2}-9 \mathrm{~N}_{2}$ mixture as propane system.

\begin{tabular}{|ccccccc|}
\hline Mixture & $\rho_{3}$ & $\rho_{4}$ & $\mathrm{u}_{3}$ & $\mathrm{u}_{4}$ & $T_{3}$ & $T_{4}$ \\
& $\mathrm{~kg} / \mathrm{m}^{3}$ & $\mathrm{~kg} / \mathrm{m}^{3}$ & $\mathrm{~m} / \mathrm{s}$ & $\mathrm{m} / \mathrm{s}$ & $K$ & $K$ \\
\hline $2 \mathrm{H}_{2}-\mathrm{O}_{2}-17 \mathrm{Ar}$ & 1.228 & 0.78 & 1107.5 & 568.4 & 1682.2 & 2760.7 \\
$2 \mathrm{H}_{2}-\mathrm{O}_{2}-5.6 \mathrm{~N}_{2}$ & 1.124 & 0.577 & 1160.9 & 506.6 & 1301.8 & 2827.7 \\
$\mathrm{C}_{3} \mathrm{H}_{8}-5 \mathrm{O}_{2}-9 \mathrm{~N}_{2}$ & 2.08 & 0.8671 & 1030 & 553.7 & 1522 & 3275.9 \\
\hline & $\mathrm{a}_{3}$ & $\theta_{\text {eff }}$ & $M_{c}$ & & & \\
& $m / s$ & $-\left(\frac{d \ln \tau_{i}}{d \ln T}\right)_{v N}$ & 19 & & & \\
\hline $2 \mathrm{H}_{2}-\mathrm{O}_{2}-17 \mathrm{Ar}$ & 784.4 & 5.4 & 0.307 & & & \\
$2 \mathrm{H}_{2}-\mathrm{O}_{2}-5.6 \mathrm{~N}_{2}$ & 800.3 & 6.9 & 0.346 & & & \\
$\mathrm{C}_{3} \mathrm{H}_{8}-5 \mathrm{O}_{2}-9 \mathrm{~N}_{2}$ & 711.2 & 12.7 & 0.256 & & & \\
\hline
\end{tabular}

Table 1: Calculated properties at the triple point contact surface for the three mixtures considered in this study. State 3 is behind the incident and transverse waves and state 4 is after reaction from state 4 ' behind the Mach stem (Schematic is shown in Fig. 1). The triple point configuration is calculated using unreactive shock polars, as discussed in the text. $\theta_{\text {eff }}$ is the effective activation energy of the mixture, based upon change in the induction time, $\tau_{i}$, in response to a change in the lead shock velocity. $\theta_{\text {eff }}$ is calculated using the Konnov kinetics mechanism ${ }^{20}$ as discussed in. ${ }^{21} M_{c}$ is the convective Mach number. Velocities $\mathrm{u}_{3}, \mathrm{u}_{4}$ and the speed of sound, $\mathrm{a}_{3}$, are in the triple point frame.

\section{Chemistry Model}

Detailed chemistry deals with a large number of single step reversible reactions; each reaction is characterized by an Arrhenius law correlating its rate of progress to the local thermodynamic state. Each rate expression is an optimized curve fit of a set of experimental data. In a computational analysis, the choice of the chemistry model is based on a trade-off between computational efficiency and accuracy. The chemistry model used in this study is the GRI model. ${ }^{22}$ The most recent release of the GRI model, version 3.0, includes 325 elementary chemical reactions and 53 species. In the latest version, propane and $\mathrm{C} 2$ oxidation products have been added. Given that the optimization of the parameters does not target fuels other than natural gas, the accuracy of the mechanism in predicting detonation wave characteristics is analyzed in this section by means of a comparison with another combustion mechanism for small hydrocarbons combustion, the Konnov mechanism. ${ }^{20}$ The Konnov mechanism includes a considerably larger number of species and reactions, 127 species and 1207 reactions. Therefore, the mechanism is optimized for a larger class of mixtures and the application target is wider. The Konnov mechanism has been previously validated for detonation temperatures and pressures by comparing the calculated ignition delay time with available shock tube data. ${ }^{21}$

The GRI mechanism is validated by comparison with the Konnov mechanism. The problem on which the GRI and Konnov mechanism are compared is a Zel'dovich-von Neumann-Döring (ZND) detonation structure supported by the hot stream injection flow of the propane system shear-layer. The results of the comparison

4 of 33

American Institute of Aeronautics and Astronautics 
are shown in Fig. 3.

The GRI and Konnov mechanisms predictions for thermo-fluid variables and species mass fractions are in reasonable agreement. Given the smaller size of the GRI 3.0 mechanism, it has been chosen for the simulations performed for this research.

\section{Linear Analysis}

The objective of the analysis presented in this section is to determine the relation between mixture type and the Kelvin-Helmholtz instability. The merit of the linear analysis in describing mixing layer instability has been shown in recent years by comparing full Navier-Stokes and linear perturbation results to experimental measurements. ${ }^{23}$ The linear analysis is relevant to this study because, due to the short induction times, any interaction between mixing and gas ignition will occur close to the triple point. The analysis here presented will focus on the relation between the chemistry and the eigenvalue growth rate.

In this analysis the mixtures, Table 1, are characterized on the basis of their effective activation energy, and are denoted as low, the hydrogen-oxygen-argon system, medium, the hydrogen-oxygen-nitrogen system, and high, the propane system, reactivity mixtures. The effective activation energy is defined as the scaled activation energy of a reduced one step mechanism associated with the post shock conditions. The correlation between transversal ignition and activation energy was suggested by Arienti and Shepherd. ${ }^{16}$ Such a categorization has the drawback of not including the effects of molecular diffusion. Mixture characterization on the basis of free (adiabatic) burning velocity rather than mixture activation energy might be considered. However, Singh et al. ${ }^{15}$ have reported about the difficulty of uniquely defining a free burning velocity for the shocked reactant, due to its high temperature. The simulations of Arienti and Shepherd ${ }^{16}$ have shown that the propagation speed of the transversal ignition front increases with the mixture activation energy for similar mixtures and conditions to those considered in this investigation.

A linear analysis of a reacting mixing layer was performed $i^{24}$ for a constant caloric properties system with simplified one step kinetics. The approach adopted in the present research is to preserve the caloric and chemical complexity of the system offering a realistic insight into the development of the instabilities.

\section{A. Mean-flow}

The mean-flow is defined by the two dimensional compressible Navier Stokes equation in the boundary layer form which neglects the pressure gradients, retains only the mixed $\sigma_{x y}$ component of the shear stress, and considers only the $y$ component of the heat flux and species diffusive fluxes. In what follows $N_{s}$ represents the number of species in the model and $N_{e l}$ is the number of atomic elements. In dimensional form:

$$
\begin{aligned}
\frac{\partial \rho u}{\partial x}+\frac{\partial \rho v}{\partial y} & =0 \\
\rho u \frac{\partial u}{\partial x}+\rho v \frac{\partial u}{\partial y} & =\frac{\partial}{\partial y}\left(\mu \frac{\partial u}{\partial y}\right) \\
\rho u \frac{\partial h}{\partial x}+\rho v \frac{\partial h}{\partial y} & =\frac{\partial}{\partial y}\left(\lambda \frac{\partial T}{\partial y}\right)+\mu\left(\frac{\partial u}{\partial y}\right)^{2} \\
\rho u \frac{\partial Y_{j}}{\partial x}+\rho v \frac{\partial Y_{j}}{\partial y} & =\sum_{k=1}^{N_{s}} \frac{\partial}{\partial y}\left(\nu_{j, k} \frac{\partial Y_{k}}{\partial y}\right)+\rho \Omega_{j}, \quad j=1, \ldots, N_{s}
\end{aligned}
$$



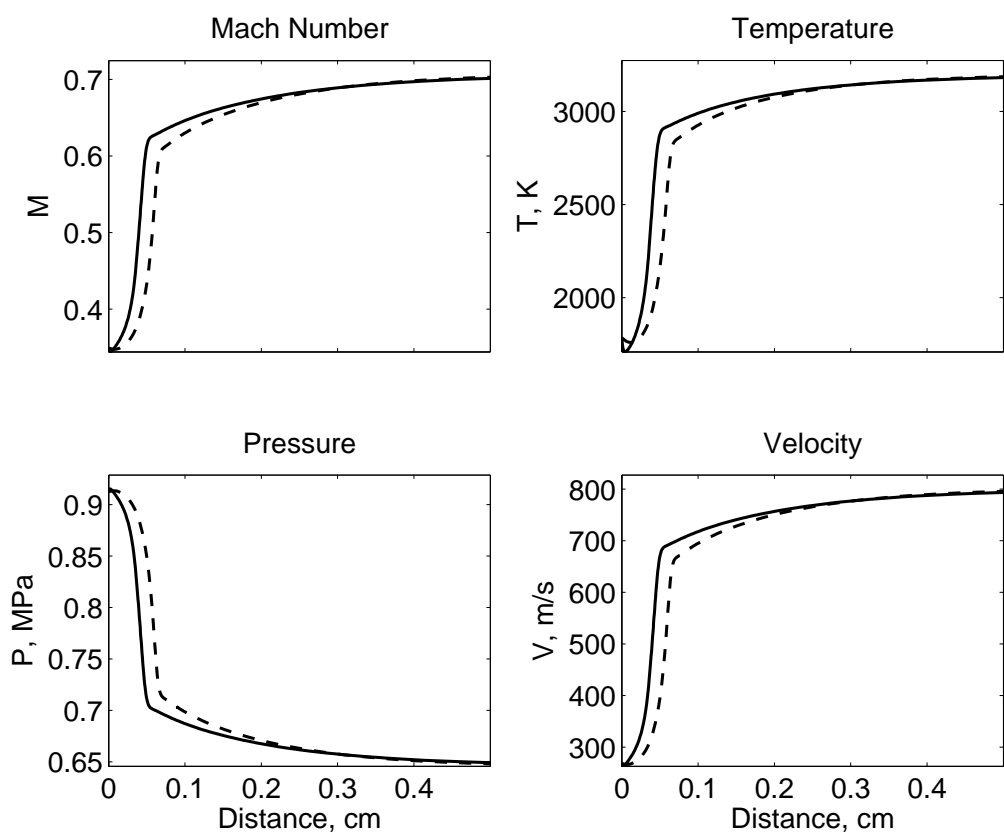

(a) Fluid dynamic variables
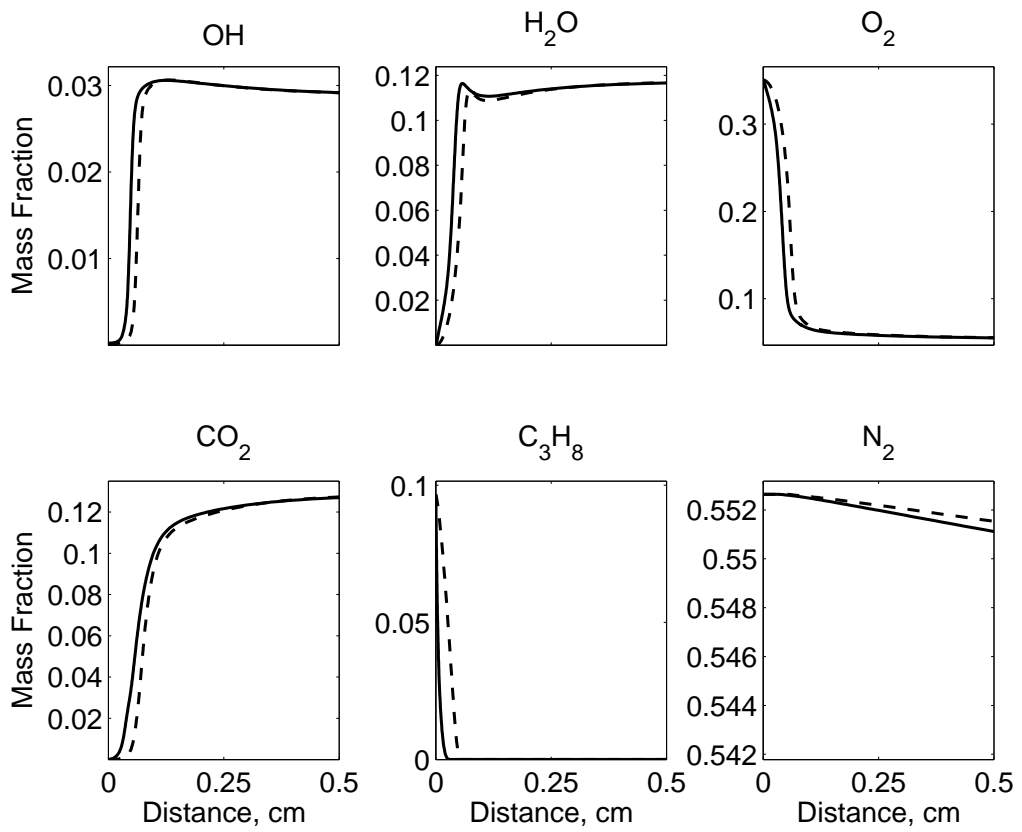

(b) Selected species mass fraction

Figure 3: A comparison between two chemistry models. Prediction of the ZND detonation profile for $\mathrm{C}_{3} \mathrm{H}_{8}-$ $5 \mathrm{O}_{2}-9 \mathrm{~N}_{2}$, initial pressure $20 \mathrm{kPa}$. Solid line, Konnov chemistry model, dashed line GRI chemistry model.

6 of 33

American Institute of Aeronautics and Astronautics 
the previous set of equations is completed by the thermal and caloric equations of state, the imposition of the constant pressure and the rate term Arrhenius approximations, $\dot{\omega}_{j}$,

$$
\begin{aligned}
& P=P_{o} \\
& T=T\left(P, h, Y_{j}\right) \\
& \rho=P /\left(T \sum_{k=1}^{N_{s}} R_{k} Y_{k}\right) \\
& \Omega_{j} \equiv \dot{\omega}_{j} / \rho=\Omega_{j}\left(P, h, Y_{k}\right), \quad j=1, \ldots, N_{s} .
\end{aligned}
$$

A solution of the mean flow equation is carried out by first introducing the Howarth-Dorodnitzyn transformation,

$$
\begin{aligned}
& \tilde{y}=\int_{0}^{y} \rho d t \\
& \tilde{v}=\rho v+u \int_{0}^{y} \rho_{x} d t
\end{aligned}
$$

which transforms the equations to

$$
\begin{aligned}
\frac{\partial u}{\partial x}+\frac{\partial \tilde{v}}{\partial \tilde{y}} & =0 \\
u \frac{\partial u}{\partial x}+\tilde{v} \frac{\partial u}{\partial \tilde{y}} & =\frac{\partial}{\partial \tilde{y}}\left(\rho \mu \frac{\partial u}{\partial \tilde{y}}\right) \\
u \frac{\partial h}{\partial x}+\tilde{v} \frac{\partial h}{\partial \tilde{y}} & =\frac{\partial}{\partial \tilde{y}}\left(\rho \lambda \frac{\partial T}{\partial \tilde{y}}\right)+\mu \rho\left(\frac{\partial u}{\partial \tilde{y}}\right)^{2} \\
u \frac{\partial Y_{j}}{\partial x}+\tilde{v} \frac{\partial Y_{j}}{\partial \tilde{y}} & =\sum_{k=1}^{N_{s}} \frac{\partial}{\partial \tilde{y}}\left(\rho \nu_{j, k} \frac{\partial Y_{k}}{\partial \tilde{y}}\right)+\Omega_{j}, \quad j=1, \ldots, N_{s} .
\end{aligned}
$$

Here $\nu_{i, k}$ are the effective ordinary multicomponent diffusion coefficients. The word effective symbolize the fact that they directly multiply the mass fraction gradients. They are related to the ordinary multicomponent diffusion coefficients, $\tilde{\nu}_{j, l}$, by

$$
\nu_{j, l}=\rho m_{j}\left(\sum_{k=1}^{N_{s}} \frac{\tilde{\nu}_{j, k}}{m_{l}}-\frac{\tilde{\nu}_{j, l}}{\hat{m}}\right), \quad j=1, \ldots, N_{s}, l=1, \ldots, N_{s},
$$

with $m_{j}$ and $\hat{m}$ the species and mixture mean molecular weights. The ordinary diffusion coefficients are obtained from the binary diffusion coefficients using the "extended" Chapman-Enskog procedure of DixonLewis. ${ }^{25}$ Likewise, the other transport coefficients, which include the mixture average thermal conductivity, $\lambda$, are based on kinetic theory approximate solutions and are determined using a CHEMKIN based multicomponent transport package. ${ }^{26,27}$

In this form, the only difference between the system composed by the momentum and continuity equation and its incompressible nonreactive analog is the dependence of the product $\rho \mu$ on the species mass fractions and temperature. To simplify the analysis, we assume that the product is constant and equal to its freestream value,

$$
\rho \mu=(\rho \mu)_{\infty},
$$

see Lock. ${ }^{28}$ This approximation leads to the solution of the velocity components in terms of the similarity variable,

$$
\eta=\left(\frac{u}{2 \rho \mu}\right)_{\infty} \frac{\tilde{y}}{\sqrt{x}}
$$

by seeking a similarity solution for which the zero (stemming from the point of initial contact) streamline is horizontal,

$$
\begin{gathered}
u=F^{\prime}(\eta) u_{\infty} . \\
7 \text { of } 33
\end{gathered}
$$


The similarity equation is

$$
F^{\prime \prime \prime}+F F^{\prime \prime}=0
$$

with boundary conditions:

$$
\begin{aligned}
& F^{\prime}(\infty)=1 \\
& F(0)=0 \\
& F^{\prime}(-\infty)=\beta_{u} \equiv u_{-\infty} / u_{\infty}
\end{aligned}
$$

The species and temperature equations are solved numerically by means of a pseudo-time marching algorithm, using the self similar velocity field previously evaluated at the mesh points. The computational mesh used for the mean flow computation is of size [200 $\times 500]$, and the solution domain is the box $\left\{(x, \eta) \mid x-x_{0} \in[0,0.5], \eta \in[-20,20]\right\}$. Here, $x_{0}$ is the distance between the inflow boundary and the detonation triple point; we take $x_{0}=0.1 \mathrm{~cm}$. For this distance, the term $\sqrt{\frac{R e x / L}{2}}$ takes values 270 for the propane system, 175 for the hydrogen-oxygen-argon system, and 214 for the hydrogen-oxygen-nitrogen system. The boundary conditions for the temperature and species fields are zero gradient boundary conditions in the cross flow direction, while the inflow boundary condition is determined by solving the nonreactive equation with constant caloric properties and unitary Prandtl and Schmidt numbers. Thermal and mass diffusivities are related to the viscosity by

$$
\frac{\rho \lambda}{c_{p}}=(\rho \mu)_{\infty}, \quad \rho \nu_{i, k}=(\rho \mu)_{\infty} \delta_{i, k},
$$

where $\delta$ is the Kronecker symbol. The following injection conditions are obtained:

$$
\begin{aligned}
T /(T)_{\infty} & =K_{1,0} F^{\prime}+K_{2,0}-\frac{(\gamma)_{\infty}-1}{2} M^{2}\left(F^{\prime}\right)^{2} \\
Y_{i} & =K_{1, i} F^{\prime}+K_{2, i}
\end{aligned}
$$

where the constants $K_{j, i}$ are obtained by satisfying the conditions at $\eta= \pm \infty$. Zero gradient boundary conditions are used for the outflow boundary.

\section{B. Mean-flow results}

The mean-flow results are shown in Fig. 4 for a distance $x-x_{0}=0.25 \mathrm{~cm}$, approximately half of the minimum value of the constant volume induction distance for the three systems, $\left(\tau_{i} u_{3}\right)_{\min }=0.58 \mathrm{~cm}$, which corresponds to the low activation energy case. The solid curves in each subfigure represent the ratios $T / T_{\infty}$, while lines of other type represent selected species mass fractions that are normalized using the values on the hot side of the shear-layer, $\eta=-\infty$. The size of the region where molecular diffusion is significant is of similar magnitude for the three mixtures and it extends to cover $\eta \in[-5,5]$. The location of the transversal ignition front is clear only for the high activation energy mixture, while for the others the region of chemical activity is spread over the mixing region. The mean-flow analysis leads to the identification of a region of dynamic shear, associated with the maximum velocity gradient and located around $\eta=0$, and a region of thermal shear associated with the temperature and species gradients and located in proximity of the transversal ignition front. A decoupling between dynamic shear region and thermal shear region is evident only for the propane system. The propane system is the only one that shows a temperature undershoot on the cold side of the shear-layer, while the two other mixtures show a temperature overshoot on the hot side of the mixing layer. In the two lower activation mixtures, there is a distinguishable overshoot in the $\mathrm{OH}$ mass fraction.

The overshoot is always positioned around the origin and is a consequence of the viscous heating. The associated bulge in $\mathrm{OH}$ concentration is in agreement with what was shown by the computations of Arienti and Shepherd, ${ }^{16}$ but here the peak increases in magnitude for the low activation energy mixture and totally disappears for the high activation energy case. The overshoot is associated with the dynamic shear rather than the ignition front and therefore it is not a factor in controlling the flame spread in the fresh mixture.

8 of 33

American Institute of Aeronautics and Astronautics 


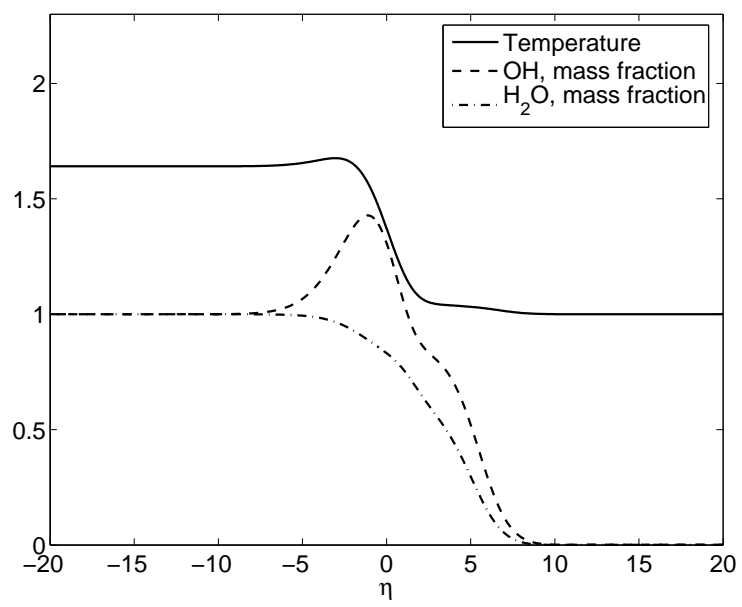

(a) hydrogen-oxygen-argon system

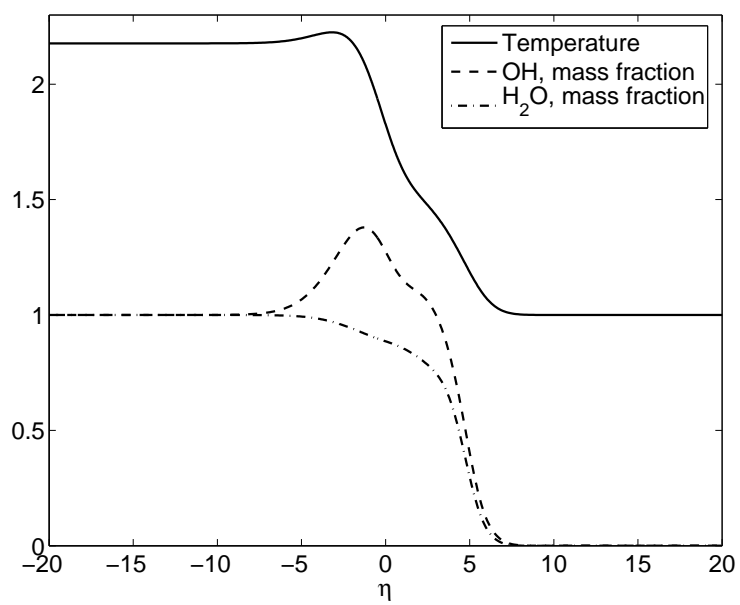

(b) hydrogen-oxygen-nitrogen system

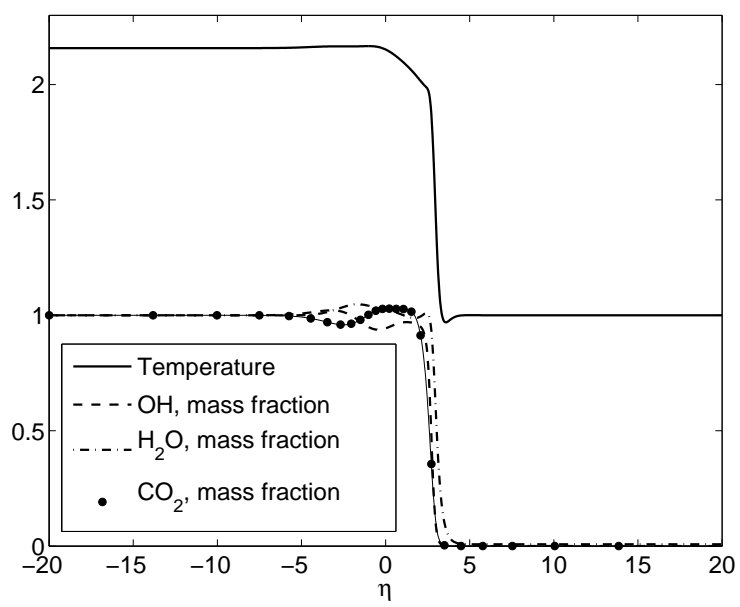

(c) propane system

9 of 33

Figure 4: Mean flow at a location $x-x_{0}=0.25 \mathrm{~cm}$, note, the mass fractions are normalized by the values on the hot side of the layer. 


\section{Inviscid perturbation}

The mean-flow profiles previously computed are used in an inviscid perturbation analysis by considering a parallel flow in chemical equilibrium and the perturbations to such equilibrium. In the reminder of this section, the variables are non-dimensionalized using as length scale, $L_{s}=\sqrt{x(2 \mu /(\rho u))_{\infty}}$. The velocity, pressure, density and temperature are non-dimensionalized by the corresponding values at $\eta=+\infty$, the enthalpy by the term $\left(c_{p} T\right)_{\infty}$, where $c_{p}$ is the mixture averaged specific heat. The species gas constants, $R_{j}$ and the specific heats are non-dimensionalized by the mixture average specific heat at plus infinity, and the ratio between velocity scale and length scale, $(u)_{\infty} / L_{s}$, is used for the reaction rates $\Omega_{j}$. The following expansions are considered:

$$
\left[u, v, p, h, \rho, Y_{j}, \Omega_{j}\right]=\left[\bar{u}, 0,1, \bar{h}, \bar{\rho}, \bar{Y}_{j}, \bar{\Omega}_{j}\right]+\left[f, \alpha \phi, \Pi, \theta, r, z_{j}, w_{j}\right] \exp (i \alpha(x-c t)) .
$$

Noticing that the prime denotes differentiation with respect to the shear-layer variable $\eta$, the equations for the perturbations are written as:

$$
\begin{array}{r}
i \frac{(\bar{u}-c)}{\bar{\rho}} r+i f+(\bar{\rho} \phi)^{\prime}=0 \\
\bar{\rho}\left[i(\bar{u}-c) f+\bar{u}^{\prime} \bar{\rho} \phi\right]+\frac{i \Pi}{\gamma_{\infty} M^{2}}=0 \\
i \alpha^{2}(\bar{u}-c) \phi+\frac{\Pi^{\prime}}{\gamma_{\infty} M^{2}}=0 \\
\bar{\rho}\left[i(\bar{u}-c) \theta+\bar{h}^{\prime} \bar{\rho} \phi\right]-i \frac{\gamma_{\infty}-1}{\gamma_{\infty}}(\bar{u}-c) \Pi=0 \\
i \alpha(\bar{u}-c) z_{j}+\bar{Y}_{j}^{\prime} \bar{\rho} \phi \alpha=w_{j}, \quad j=1, \ldots, N_{s}
\end{array}
$$

The perturbations are correlated through a set of algebraic relations dependent on the chemistry model,

$$
\begin{gathered}
r=\rho \Pi+\rho^{2} \frac{\gamma_{\infty}}{\gamma_{\infty}-1} \sum_{k=1}^{N_{s}}\left(\frac{R}{c_{p}} \bar{h}_{k}-R_{k} T\right) z_{k}-\frac{\rho}{c_{p} T} \theta \\
{\left[w_{1}, w_{2}, \ldots, w_{N_{s}-1}, w_{N_{s}}\right]^{T}=\Omega_{p} \Pi+\Omega_{h} \theta+\Omega_{y}\left[z_{1}, z_{2}, \ldots, z_{N_{s}-1}, z_{N_{s}}\right]^{T}}
\end{gathered}
$$

where $\Omega_{p}$ and $\Omega_{h}$ are vectors of size $N_{s}$, while $\Omega_{y}$ is a Jacobian matrix of size $N_{s} \times N_{s}$ all of which are obtained using the GRI chemistry mechanism.

\section{Solution of the perturbation equation}

As in the case of a non-reactive mixture, the eigenvalue problem associated with the amplification of inviscid perturbations leads to a second order homogeneous equation for the pressure perturbation,

$$
\Pi^{\prime \prime}+B \Pi^{\prime}+C \Pi=0
$$

The coefficients are described in terms of the vectors:

$$
\begin{aligned}
S_{1} & =\left[i \alpha(\bar{u}-c) \mathcal{I}-\Omega_{y}\right]^{-1}\left(-\alpha\left[\bar{Y}_{1}^{\prime}, \bar{Y}_{2}^{\prime}, \ldots, \bar{Y}_{N_{s}-1}^{\prime}, \bar{Y}_{N_{s}}^{\prime}\right]^{T}\right) \\
S_{2} & =\left[i \alpha(\bar{u}-c) \mathcal{I}-\Omega_{y}\right]^{-1} \Omega_{p} \\
S_{3} & =\left[i \alpha(\bar{u}-c) \mathcal{I}-\Omega_{y}\right]^{-1} \Omega_{h}
\end{aligned}
$$


and the coefficients

$$
\begin{aligned}
s_{j} & =\rho^{2} \frac{\gamma_{\infty}}{\gamma_{\infty}-1} \sum_{k=1}^{N_{s}}\left(\frac{R}{c_{p}} \bar{h}_{k}-R_{k} T\right) S_{j, k}+t_{j} ; \quad j=1,2,3 \\
t & =\left[\begin{array}{c}
0 \\
\bar{\rho} \\
-\frac{\bar{\rho}}{c_{p} T}
\end{array}\right]
\end{aligned}
$$

where $\mathcal{I}$ is the identity matrix. Thus,

$$
\begin{array}{r}
B=-\left[\frac{s_{3} \bar{h}^{\prime}-\bar{\rho}^{\prime}}{\bar{\rho}}+2 \frac{\bar{u}^{\prime}}{\bar{u}-c}-i s_{1} \frac{\bar{u}-c}{\bar{\rho}}\right] \\
C=-\frac{\alpha^{2}}{\bar{\rho}^{2}}\left[1-M^{2}(\bar{u}-c)^{2}\left(s_{2} \gamma_{\infty}+\frac{s_{3}}{\bar{\rho}}\left(\gamma_{\infty}-1\right)\right)\right] .
\end{array}
$$

Introducing the Riccati variable

$$
G \equiv \frac{\Pi^{\prime}}{\Pi \alpha \bar{T}},
$$

the second order equation is reduced to a first order non-linear equation

$$
G^{\prime}+\alpha \bar{T} G^{2}+\left(\frac{\bar{T}^{\prime}}{\bar{T}}+B\right) G+\frac{C}{\alpha \bar{T}}=0 .
$$

The boundary conditions are obtained by analyzing the asymptotic trend of $\Pi$ in the freestream. Noting that the coefficient B vanishes in either sides of the freestream, appropriate boundary conditions for the pressure perturbation are:

$$
\eta \rightarrow \pm \infty, \Pi \rightarrow K_{ \pm \infty} \exp (\mp \sqrt{-C \eta}) .
$$

Boundary conditions for $G$ on both sides of the free-stream are obtained by using equations (32) and (30).

\section{Numerical algorithm}

The Riccati equation is integrated in the interval $\eta \in[-20,20]$, using a $(7,8)$ order variable step (adaptive) Runge Kutta method, with an error tolerance of $1 \times 10^{-10} .{ }^{29}$ A sensitivity study to the size of the domain reveals that doubling the solution interval yields a maximum change of the "normalized" pressure perturbation eigenfunction lower than $1 \times 10^{-9}$ for $\eta \in[-20,20]$, and for all the cases discusses in the following sections. A quintic natural spline representation of the mean-flow is used, so that locally in the interval between the knots $i$ and $i+1$ a mean flow variable $\bar{v}$ is related to $p \equiv \eta-\eta_{i}$ through the quintic polynomial:

$$
\bar{v}-\bar{v}_{i}=\left(\left(\left(\left(F_{i} p+E_{i}\right) p+D_{i}\right) p+C_{i}\right) p+B_{i}\right) p
$$

where the coefficients $B_{i}, C_{i}, D_{i}, E_{i}, F_{i}$ are obtained enforcing continuity in the derivatives (up to the fourth order) at the knots. With the grid considered in this study, the error associated with the spline representation of the mean flow was shown to be small when the algorithm was tested on a mean profile where the velocity is defined by a hyperbolic tangent relation and the temperature and species by equation (13). The complex Muller method is used to find the zero of the matching condition resulting by integrating the equation (31) between $\eta \in[-20,0]$ and $\eta \in[20,0]$. A spatial stability study is pursued so that perturbation are allowed to grow in space but not in time. The eigenvalues are found for time frequencies $\omega=\alpha c$ on the real axis. 


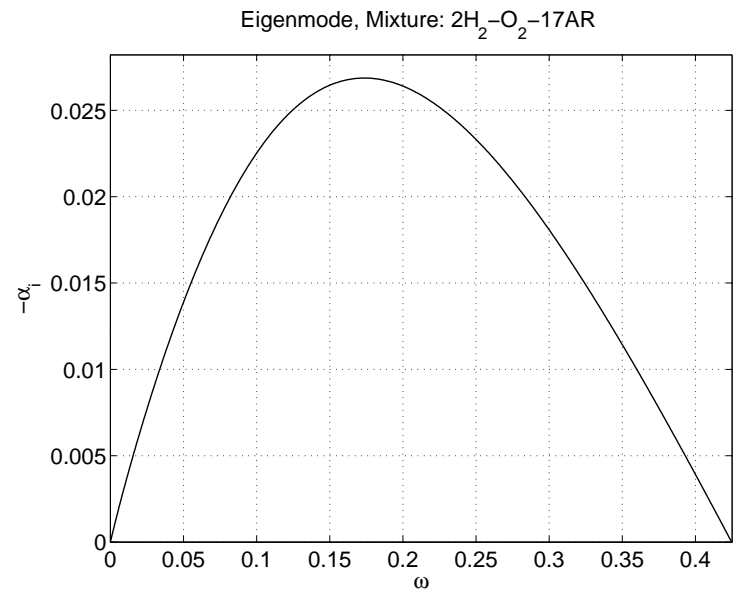

(a) hydrogen-oxygen-argon system

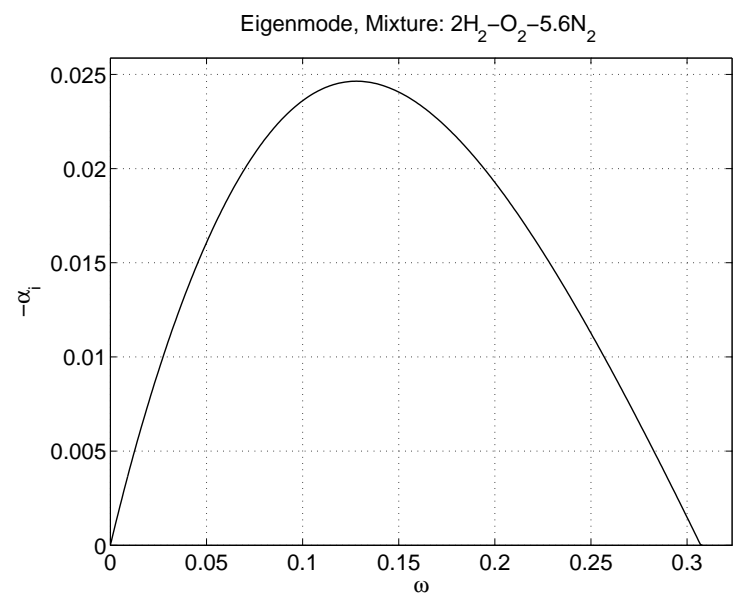

(b) hydrogen-oxygen-nitrogen system

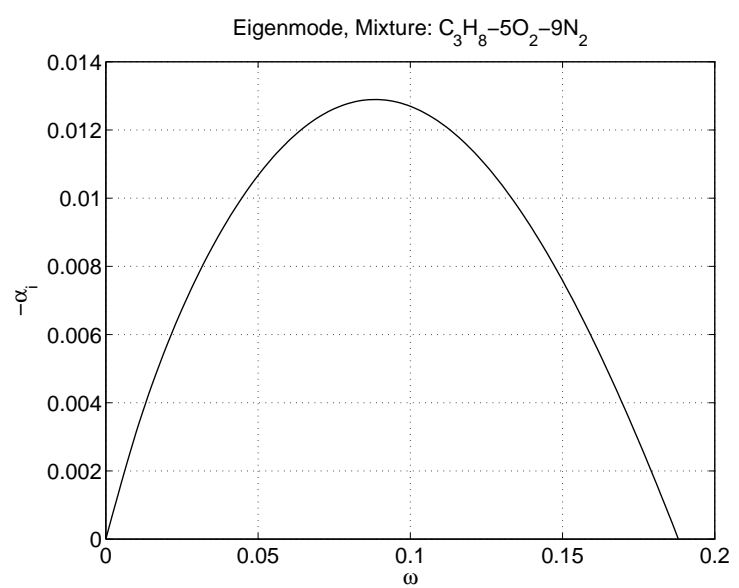

(c) propane system

12 of 33

Figure 5: Spatial growth rate of inviscid perturbation to the mean flow for the three systems. American Institute of Aeronautics and Astronautics 


\section{E. Spatial growth rate}

Only one unstable eigenmode was found for each of the three mixtures considered. The non-dimensional growth rate, $-\alpha_{i}$ is plotted versus $\omega$ for the three mixtures in Fig. 5. The set of figures shows that the disturbance growth rate is smallest for the propane system and largest for the hydrogen-oxygen-argon system. To isolate the consequences of changing the caloric versus chemical characteristics of the system, the effects of non-chemistry-related variables, such as the convective Mach number and the velocity and temperature ratios across the layer, are taken into account by comparing the growth rate to that of a frozen flow shear-layer with the chemical composition of the fresh mixture. For this comparison, the frozen mean velocity is found using the Blasius profile, while a self-similar relation is used for the mean temperature, equation (13). The caloric and thermal properties of the mixture are kept constant and identical to their frozen state. Note that Jackson and Grosch ${ }^{30}$ have proven that the use of a rheological equation other than the Lock approximation equation (7), has a negligible effect on the normalized growth rate. Analogously, based upon frozen flow eigenvalues computations, the effect of the Prandtl number is assumed not important for the range of values that correspond to the three systems here described: The frozen flow stability is carried with $\operatorname{Pr}=1$ for all the mixtures. The growth rate for the reactive computations is scaled by the corresponding maximum value for frozen chemistry, $\left(-\alpha_{i, 0}\right)_{\max }$, and plotted versus the ratio $\omega / \omega_{N}$, where $\omega_{N}$ represents the neutral frequency of the frozen modes. The results are shown in Fig. 6 . The growth rates show that chemistry has a strong effect in reducing the instability in reactive computations, both as it regards the maximum growth rate and the range of frequency of the unstable modes, when compared to the corresponding frozen case. In the next section the eigenfunctions will be presented, then, the relation between chemistry and modes spatial growth will be further elucidated by looking at the effect of finite rate chemistry in the perturbation equation.

\section{F. Eigenfunctions}

The eigenfunctions for the most unstable modes, maximum value of $-\alpha_{i}$, are plotted in this section for the three mixture. The eigenfunctions are normalized according to the condition, $\Pi(0)=1$. Fig. 7 shows the eigenfunctions for the three mixtures in terms of $\Pi$ and $\alpha \phi$. An increase in mixture activation leads to noticeable changes in the eigenfunction behavior. The eigenfunctions for the low activation energy system differ slightly from those for a frozen flow. The increase in activation energy causes the real part of the pressure perturbation to become asymmetric with the cold side becoming the stronger side. In the same way, the magnitude of the normal velocity perturbation increases with an increase in activation energy. The low and medium activation energy cases differ slightly. The fresh side, $\eta>0$, eigenfunctions for the high activation energy case differ significantly from the two other cases. The region where anomalous behavior is observed centers around $\eta=5$, such a location corresponds to the transversal ignition front position, as shown in Fig. 4(c).

\section{G. Finite rate chemistry effect}

The importance of the finite rate chemistry in the perturbation equation are determined by investigating the growth rate and the eigenfunctions for spatial evolution controlled by the frozen flow perturbation equations, and a mean flow defined by the reactive Navier-Stokes equations, equation (2). This analysis is restricted to the propane system. For frozen flow, the pressure perturbation equation coefficients analogous to those in equation (29) are:

$$
\begin{aligned}
B & =-2 \frac{\bar{u}^{\prime}}{\bar{u}-c} \\
C & =-\alpha^{2} \bar{T}^{2}\left[1-M^{2}(\bar{u}-c)^{2} / \bar{T}\right] .
\end{aligned}
$$

A comparison between the growth rate for nonreactive and reactive perturbation is shown in Fig. 8(a). The figure shows that chemical reaction in the perturbation evolution equation has a negligible effect on the 


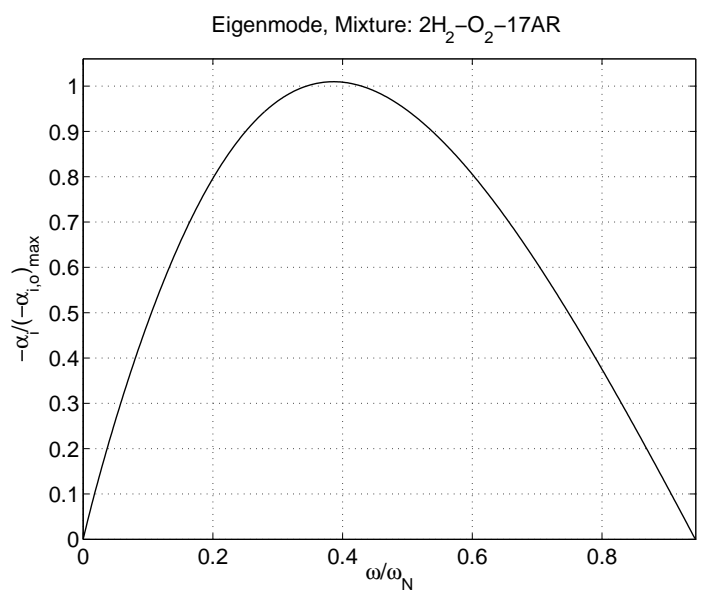

(a) hydrogen-oxygen-argon system

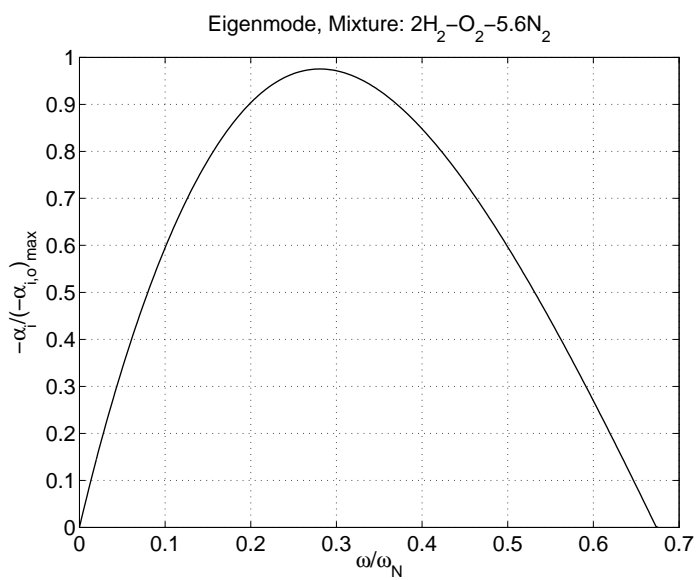

(b) hydrogen-oxygen-nitrogen system

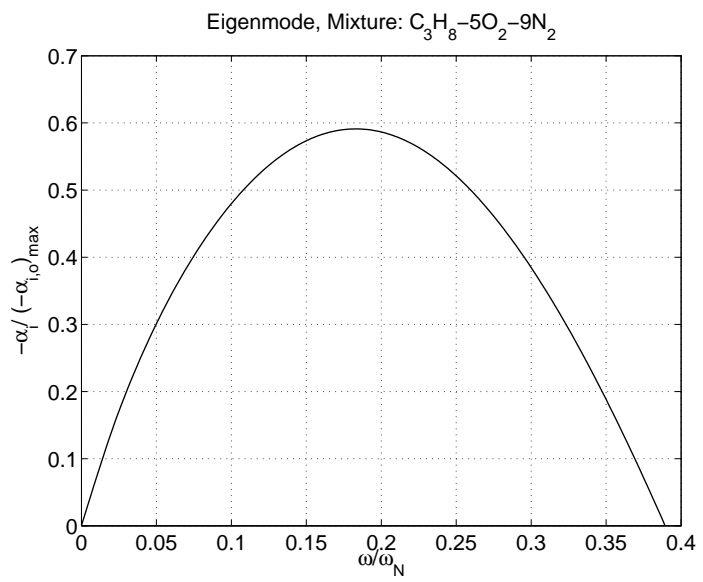

(c) propane system

Figure 6: Comparison of the spatial stability eigensabse with that of frozen systems having comparable fluid-dynamic parameters.

American Institute of Aeronautics and Astronautics 

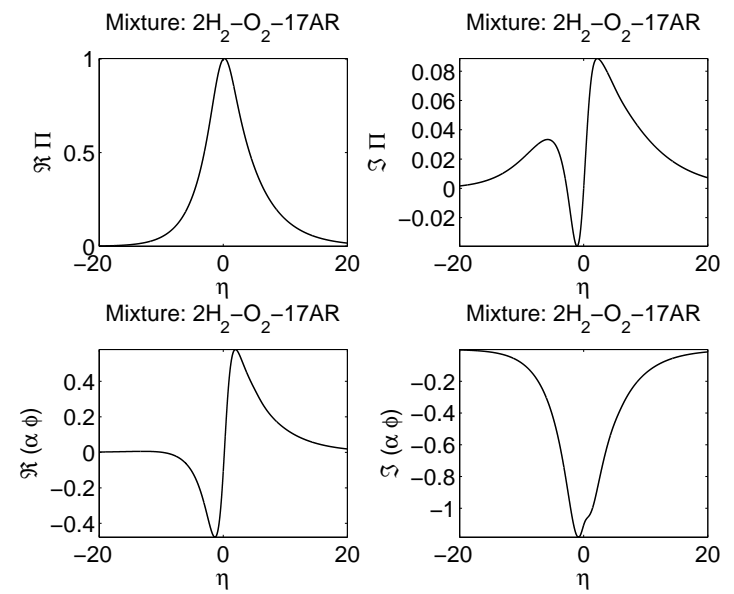

(a) hydrogen-oxygen-argon system
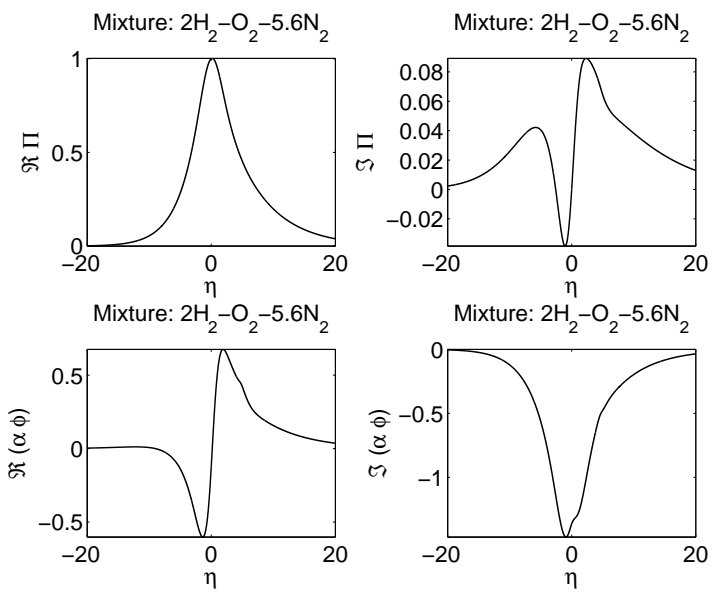

(b) hydrogen-oxygen-nitrogen system
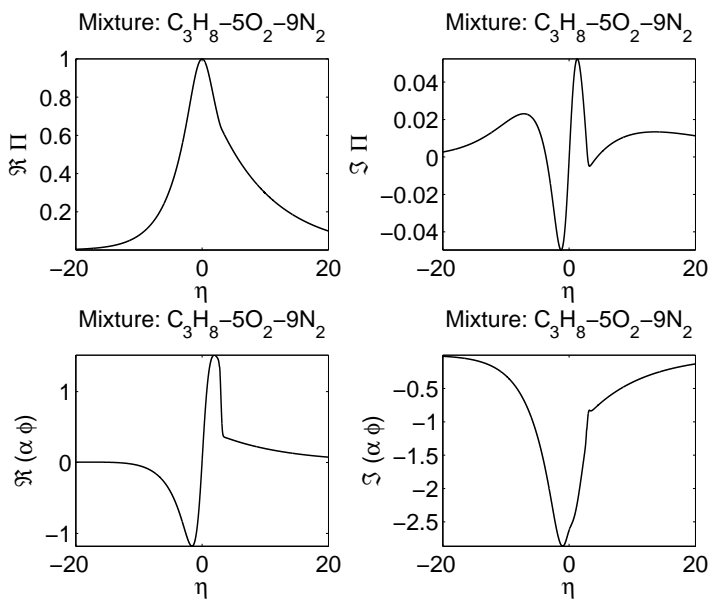

(c) propane system

15 of 33

Figure 7: Pressure and velocity eigenfunctions. American Institute of Aeronautics and Astronautics 


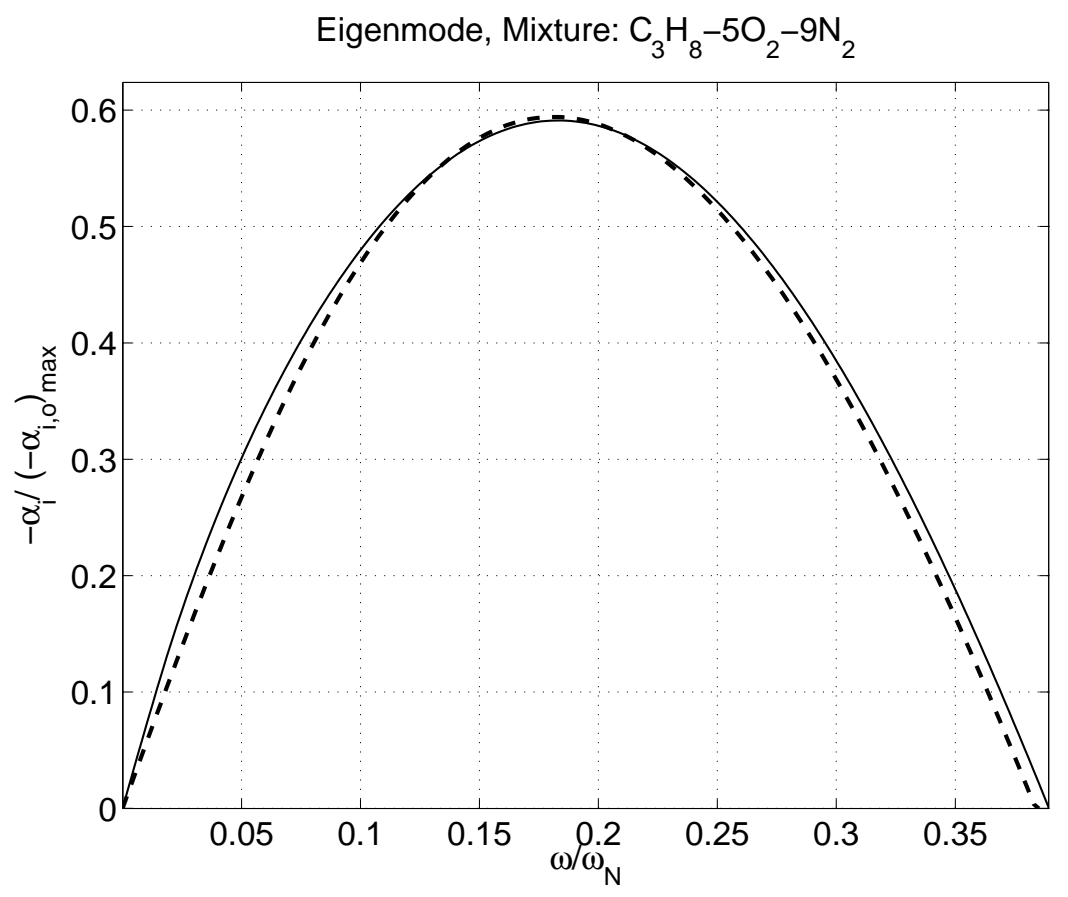

(a) Spatial growth rate
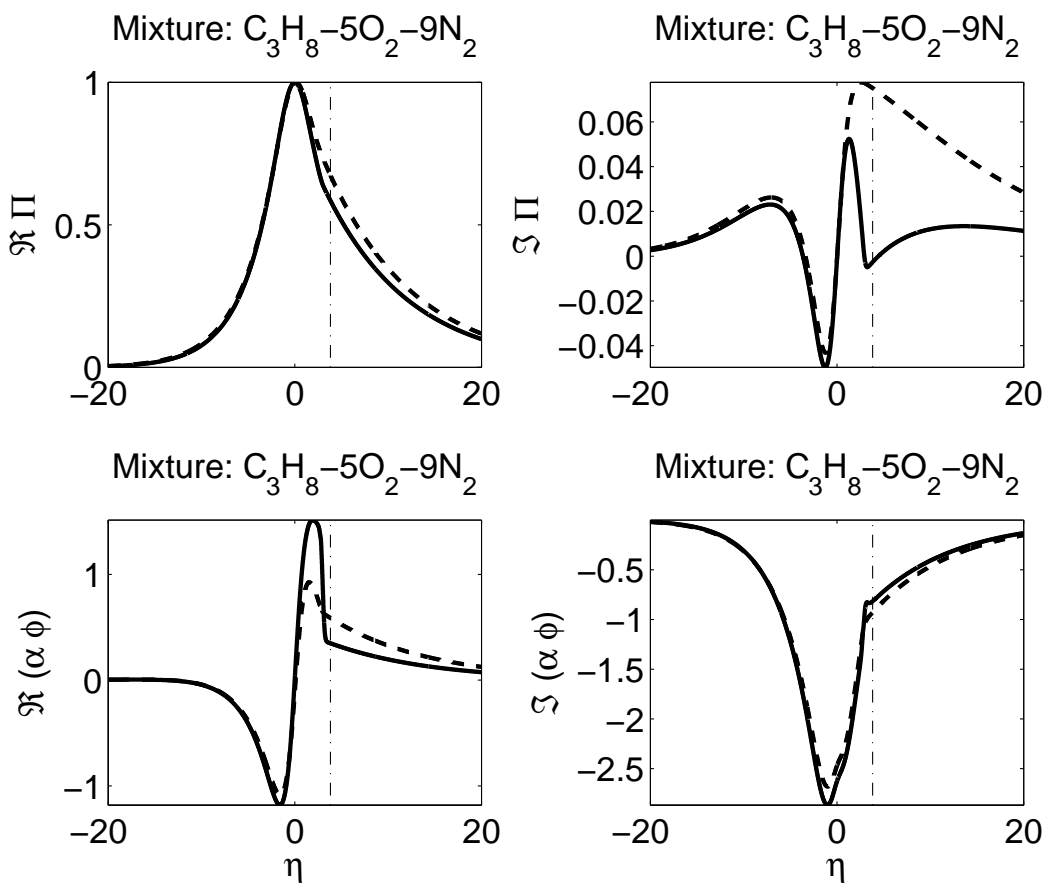

(b) Eigenfunctions

Figure 8: Solutions of frozen and reactive perturbation evolution equations for a given mean flow. The solid line is the reactive case while the dashed line is the frozen case. The vertical dash-dotted line in the eigenfunctions subfigure represents the location of the maximum mean-flow temperature derivative, located at $\eta=3.82$.

American Institute of Aeronautics and Astronautics 
growth rate. This observation leaves the mean-flow as the principal factor in determining the linear growth rate attenuation linked to an increase in effective activation energy.

The eigenfunctions that correspond to the maximum growth rate are shown in Fig. 8(b). Frozen and reactive eigenfunctions are compared. The location of the ignition front is identified as that of the maximum mean-flow temperature derivative and is shown by the dash-dotted vertical lines in the four panels of Fig. 8(b). The frozen eigenfunctions are similar to those for the hydrogen-oxygen-nitrogen system shown in Fig. 7(b). In the propane systemmixture, chemistry effects have a strong influence on the eigenfunctions. The behavior near the ignition front, $\eta \approx 5$, characteristic of the high activation energy system in Fig. $7(\mathrm{c})$, disappears in the frozen case. Moreover, the eigenfunctions for the frozen and reactive cases are almost indistinguishable in the hot side of the shear-layer, where chemical equilibrium is reached. Due to the large degree of separation of time-scale in hydrocarbon detonation systems, ${ }^{31}$ the extra dimensions of the perturbation solution for a chemical reacting system are important only in the narrow region across the ignition front. The effect of varying caloric properties of the mixture, such as the specific heats, is found to be much smaller than the contribution of the chemical source terms.

The real part of the velocity fluctuation is considerably larger in the cold (fresh) stream for the reactive calculation as compared to the frozen chemistry calculation. The real part of the pressure fluctuation show that the asymmetric pressure perturbation profile is maintained by the frozen dynamics, suggesting that this effect is also associated with the mean flow.

The linear analysis leads us to the conclusion that the correlation between growth rate reduction and effective activation energy centers around the decoupling between dynamic shear and thermal gradient in the mean profile. Such decoupling is associated with the transversal ignition front propagation in the premixed mixture, so that, for highly reactive mixtures, the region of high thermal gradient is significantly displaced towards the cold stream. Overall this effect should be associated with both diffusion and mixture effective activation energy.

Day et al. ${ }^{32}$ related the attenuation of the disturbance growth in diffusion controlled, reactive mixing layers to the reduction of the density weighted vorticity, $\bar{\rho} d \bar{u} /\left.d \eta\right|_{\eta=0}$. It should be noted that Day et al. ${ }^{32}$ used a flame sheet model so that the effect of the reactivity was not included. For the triple point shearlayer, the value of density weighted vorticity is decreased by an increase in the steepness of the ignition front and therefore, for the cases examined here, a correlation between $\bar{\rho} d \bar{u} /\left.d \eta\right|_{\eta=0}$ and mixture activation energy can be established. Thus, the mechanism of spatial growth attenuation in triple point detonation shear-layers is similar to those described in earlier work on diffusion limited combustion systems, but here the mixture activation energy is a controlling factor. A decoupling between dynamic and thermal gradients is achieved only for high activation energy mixture; the medium and low reactivity cases have very similar shear-layer characteristics even though their mixture properties are very different. These concepts will be elaborated on by performing 2-dimensional Navier-Stokes simulations of the systems, where the relation between shear-layer instability and thermal runaway in the premixed streams will be the focal point. The goal is to investigate the cause of the localized explosions noted in the experiments.

\section{Non linear numerical analysis}

The non linear analysis is carried out through numerical solutions of the two-dimensional Navier-Stokes equation. The goal of the analysis is to determine the role of the Kelvin-Helmholtz instability and the diffusion controlled combustion wave in the formation of localized explosion in the fresh mixtures.

The set of governing equations is

$$
\frac{\partial \vec{U}}{\partial t}+\frac{\partial \vec{F}}{\partial x}+\frac{\partial \vec{G}}{\partial y}=\dot{\omega}
$$


where:

$$
\vec{U}=\left[\begin{array}{l}
\rho \\
\rho u \\
\rho v \\
\rho e_{0} \\
\rho Y_{i}
\end{array}\right], \quad \vec{F}=\left[\begin{array}{l}
\rho u \\
\rho u^{2}-\sigma_{x x} \\
\rho u v-\sigma_{y x} \\
\left(\rho e_{0}-\sigma_{x x}\right) u-\sigma_{x y} v-\lambda \frac{\partial T}{\partial x} \\
\rho u Y_{i}+\sum_{k} \nu_{i, k} \frac{\partial Y_{k}}{\partial x}
\end{array}\right], \quad \vec{G}=\left[\begin{array}{l}
\rho v \\
\rho u v-\sigma_{x y} \\
\rho v^{2}-\sigma_{y y} \\
\left(\rho e_{0}-\sigma_{y y}\right) v-\sigma_{y x} u-\lambda \frac{\partial T}{\partial y} \\
\rho v Y_{i}-\sum_{k} \nu_{i, k} \frac{\partial Y_{k}}{\partial y} .
\end{array}\right] .
$$

Here $\sigma$ is the viscous stress tensor. Unlike in the linear analysis, the mixture average shear viscosity is found by using kinetic theory approximate solution as for the other transport coefficients.

The Navier-Stokes equation are discretized using a third order in time, fourth order in space Rusanov Kutler Lomax and Warming (RKLW) scheme described in Kennedy and Carpenter. ${ }^{33,34}$ The fourth order scheme is stable with a maximum CFL condition of 1.34 for linear constant coefficients scalar equations in one dimension. The RKLW is not monotone or total variation diminishing (TVD), thus in order to preserve stability for non linear or variable coefficient equations, linear filtering is used to damp out spurious oscillations. A fourth order explicit filter, also discussed by Kennedy and Carpenter, ${ }^{33}$ is used.

Stiff chemistry is handled using a fractional step splitting technique. The splitting considers the time integration of the transport and kinetic parts of the NS equations separately. The splitting scheme preserves the spatial order of the discretization, but lower the temporal order of the RKLW producing a second order time discretization. The system of governing equations is written in the operator form,

$$
\frac{\partial \vec{Q}}{\partial t}=\mathcal{T} \vec{Q}+\mathcal{K} \vec{Q}
$$

where the field variable is $\vec{Q}$, and $\mathcal{T}$ and $\mathcal{K}$ are the transport and kinetic operators, respectively. Consider the two discrete solution operators, $\mathcal{T}^{n}(\Delta t)$ and $\mathcal{K}^{n}(\Delta t)$ which approximate the solutions at time $t_{0}+\Delta t$ to the problems in which only one operator at the time is considered. The overall update is

$$
\vec{Q}^{n+1}=\mathcal{T}^{n}\left(\frac{\Delta t}{2}\right) \mathcal{K}^{n+1 / 2}(\Delta t) \mathcal{T}^{n+1}\left(\frac{\Delta t}{2}\right) \vec{Q}^{n} .
$$

Assuming that both $\mathcal{T}^{n}(\Delta t)$ and $\mathcal{K}^{n}(\Delta t)$ yield an approximation of order $(2,4)$ to the partial problems, the numerical solution to the Navier-Stokes equations is accurate of order $(2,4)$. The kinetic update is a point-implicit operation and is discretized using the Crank-Nicholson formula. The kinetic update involves progress along the reaction paths as well as the imposition of elemental mass conservation. Given that the latter constitutes a linear time-independent coefficient system, the associated matrix is inverted as a pre-process step. Chemistry update via the Crank-Nicholson method involves, therefore, the solution of a $N_{s p}-N_{e l}$ system of equations, where $N_{e l}$ is the number of atomic elements.

\section{A. Boundary conditions}

Inflow boundary conditions for the non linear analysis are found from the exact solution for the nonreactive boundary layer equations in self similar form, equation (13). The separation distance from the point of first mixing is assumed to be $x_{0}=0.1 \mathrm{~cm}$. The boundary conditions are enforced in the form of characteristic variable boundary conditions, based on the approach detailed by Poinsot and Lele. ${ }^{35}$ Boundary conditions are imposed on the primitive variable vector $\vec{P}=\left[P, u, v, T, Y_{i}\right]^{T}$ in the form

$$
\frac{\partial \vec{P}}{\partial t}=\mathcal{P} \frac{\partial \vec{Q}}{\partial t}=k_{b}\left(\overrightarrow{P_{b}}(t)-\vec{P}\right)
$$

where the matrix $\mathcal{P}$ is a rectangular matrix of size $\left[m \times N_{s p}+4\right]$ having as rows those of the Jacobian $\frac{\partial \vec{P}}{\partial \vec{Q}}$ corresponding to the $m$ imposed primitive variables $\vec{P}_{b}$. Considering a boundary with constant streamwise 
coordinate, $x$, multiplying the Navier Stokes equations by $\mathcal{P}$, one gets

$$
k_{b}\left(\vec{P}_{b}-\vec{P}\right)=-\mathcal{P}\left(\frac{\partial \vec{F}}{\partial x}+\frac{\partial \vec{G}}{\partial y}-\dot{\omega}\right)
$$

and neglecting the viscous fluxes in the direction normal to the boundary, a spectral decomposition of the flux Jacobian yields

$$
\frac{\partial \vec{F}}{\partial x}=R^{-1} \Lambda R \frac{\partial \vec{Q}}{\partial x}=R^{-1} \mathcal{L}
$$

Substituting equation (41) into equation (40) one obtains a linear system with associated coefficient matrix of size $m \times N_{s p}+4$ in the unknown vector $\mathcal{L}$. The coefficient matrix is $\mathcal{M}=\mathcal{P} R^{-1}$. This system is augmented with the evaluation of the wave amplitudes, elements of $\mathcal{L}$, associated with outgoing waves using one sided third order finite difference approximation about the boundary point. Once the wave amplitudes are solved for, equation (41) is used to evaluate the fluxes normal to the boundary. For non-reflecting boundary conditions the columns of $\mathcal{M}$ corresponding to the outgoing waves are zeroed.

For the simulations presented below, reflecting boundary conditions are applied to the inflow, where all the primitive variables except pressure on the subsonic side are imposed, and non reflecting boundary conditions with imposed pressure applied to the outflow boundaries and the side boundaries. The injection conditions are perturbed by adding a white noise, uniformly random and uncorrelated disturbance, on the imposed streamwise velocity. The magnitude of the disturbance has been selected at $4 \%$ of the nominal injection value. A set of runs with different values of white noise magnitude have revealed negligible differences in the results presented at the end of this section.

For a non reflective boundary condition, the constant $k_{b}$ in equation (40), represents an elastic response of the outside system to perturbation of the boundary values. The term non-reflective indicates that the response of the system to outgoing waves is neglected. When the constant $k_{b}$ is set equal to zero a perfectly non reflective boundary conditions in the sense of Poinsot and Lele, ${ }^{35}$ is recovered.

\section{B. One-dimensional analysis of the cold/supersonic stream}

An Euler 1D reactive analysis for the cold stream is of interest because the associated injection conditions are supersonic and therefore a classical ZND detonation structure is not supported. A ZND analysis leads to the singular condition Mach $=1$ in the cold stream before equilibrium is reached. This outcome is a necessary consequence of the nonexistence of equilibrium solutions for the given (injection) values of mass flow, momentum flux, total enthalpy and atomic fluxes, $(\rho u)_{0},\left(p+\rho u^{2}\right)_{0}, h_{0}^{o}$ and $\dot{N}_{O, H, C, N} \equiv \rho u N_{O, H, C, N}$. Equilibrium one-dimensional non-diffusive solutions are determined from the relations,

$$
\begin{aligned}
h^{o} & =\left(h^{o}\right)_{0} \\
\rho u & =(\rho u)_{0} \\
P+\rho u^{2} & =\left(P+\rho u^{2}\right)_{0} \\
\dot{N}_{O, H, C, N} & =\left(\dot{N}_{O, H, C, N}\right)_{0} \\
\dot{w}_{i=1: N_{s}-N_{e l}} & =0 .
\end{aligned}
$$

In Fig. 9(a,c,e) the equilibrium values for pressure and Mach number are plotted against the momentum flux for the three mixtures. The lines correspond to the case for which the total enthalpy, mass flux and the molar atomic fluxes are equal to their nominal (experiment/shock-polar) values, referred to as "State 3 " as in the triple point shock-polar. The vertical dashed lines indicate the nominal momentum flux. The lack of an equilibrium solution is associated with the presence of a turning point in correspondence of the sonic condition ${ }^{\mathrm{a}}$, so that a limiting value of the momentum flux is defined for each system. A similar

a The turning point is not located exactly at the sonic point, indeed this would be true for a frozen flow configuration. For a reactive flow the species mass fraction differential are proportional to the pressure differential and the $M a c h=1$ condition does not exactly define the turning point. 
argument is provided by the $P-V$ diagrams presented in Fig. 9(b,d,f). In these subfigures the Rayleigh lines emanating from "State 3", $P-P_{3}=(\rho u)_{0}^{2}\left(V_{3}-V\right)$, and the Hugoniot curve for the equilibrium products, $h-h_{3}=1 / 2\left(P-P_{3}\right)\left(V+V_{3}\right)$, are shown. The lack of an equilibrium point is demonstrated by the two curves not having an intersection point.

It is remarked that this conclusion is valid only for a one-dimensional problem, which is relevant to the shear-layer system if the curvature of the leading shock can be neglected. Experimental schlieren images show that the curvature of the leading shocks in the vicinity of the triple point is quite small near the end of the cell cycle. If the curvature is neglected and in absence of normal expansion of the stream, the large time solution away from the mixing region is a steady one-dimensional combustion wave. Stream tube divergence can facilitate the formation of stationary solutions in the natural (shock) reference frame. Assuming a quasi-one-dimensional flow, the pressure differential is given by

$$
d p=\frac{\rho u^{2}}{1-M^{2}}\left[d(\log A)+\sum_{i}^{N_{s}}\left(\frac{h_{i}}{c_{p} T}-\frac{R_{i}}{R}\right) d Y_{i}\right],
$$

from which it is seen that stream tube divergence represented by the term $d(\log A)$ can make the pressure derivative finite even when the thermicity, the second term in the square brackets, does not vanish at the sonic condition.

For a free shear-layer, expansion of the stream-tubes has to be considered. The relation between volume dilatation and stream-tube section expansion in the region of high chemical activity is of key importance in the context of premixed streams shear-layers. For a constant pressure analysis, the area expansion and the dilatation field are of the same order of magnitude. In order to account for the stress-free nature of the side wall boundaries, the parameter $k_{b}$ in equation (39) for the side boundary conditions has been increased from zero in order to simulate the response of the outer system to explosion in the streams. A value of $k_{b}=100$ is sufficient for the hydrogen-oxygen-argon system shear-layer to reach a quasi steady 2D solution in the region away from the mixing. For the two other systems it is not possible to stabilize the flow by choosing the value of $k_{b}$.

Although the interaction between the shear-layer and the outer-system is likely to be more complex when the entire detonation structure is considered, the simple boundary condition model provides a way to quantify the level of interaction between free-stream and core flow. The fact that only for the hydrogen-oxygen-argon system the flow can be stabilized to form a regular explosion profile offers an explanation for the formation of localized, two-dimensional explosions structures observed in high $\theta_{\text {eff }}$ mixtures. The explanation centers around the impossibility of obtaining an equilibrium solution in the shock frame. Experiments and large scale simulations, discussed in the next section, have shown "hot spot" formation for the high effective activation energy mixtures. The one dimensional analysis links this outcome to the inability of the stream-tube crosssectional area dilatation to overcome the large volume increase due to reaction.

Another possible explanation for the localized luminescent regions observed in the experiments is the interaction between the chemical processes and the fluid-dynamic instability based on the convective thermal transport due to roll-ups. The contribution of fluid entrainment to the "hot spot" formation will be analyzed in the next section.

\section{Results}

\section{Computational mesh}

The 2 dimensional computational meshes are uniformly spaced in the streamwise direction with spacing $d x=12.5 \mu \mathrm{m}$ and exponentially spaced in the direction perpendicular to the flow with minimum spacing $d y_{\text {min }}=1.7 \mu \mathrm{m}$ and average spacing $d y_{\text {avg }}=20 \mu \mathrm{m}$. Using the length scale defined in the linear analysis section with $x=0.5 \mathrm{~cm}$, the minimum spacing for the hydrogen-oxygen-argon system is, in non-dimensional units, $d \bar{y}_{\min }=7 \times 10^{-2}$.

A grid convergence study was performed by decreasing the spacings in both direction by a factor 1.5 . The results of the grid refinement study are shown in Fig. 10. The grid on which numerical experiments 


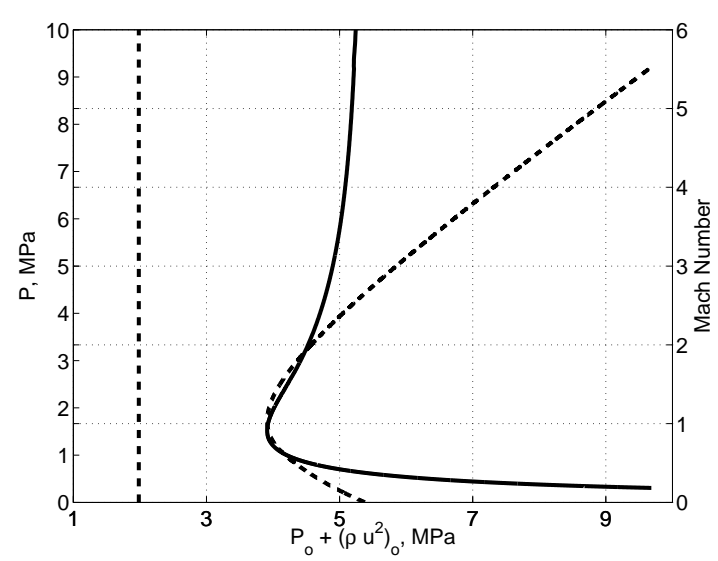

(a) hydrogen-oxygen-argon system, solutions.

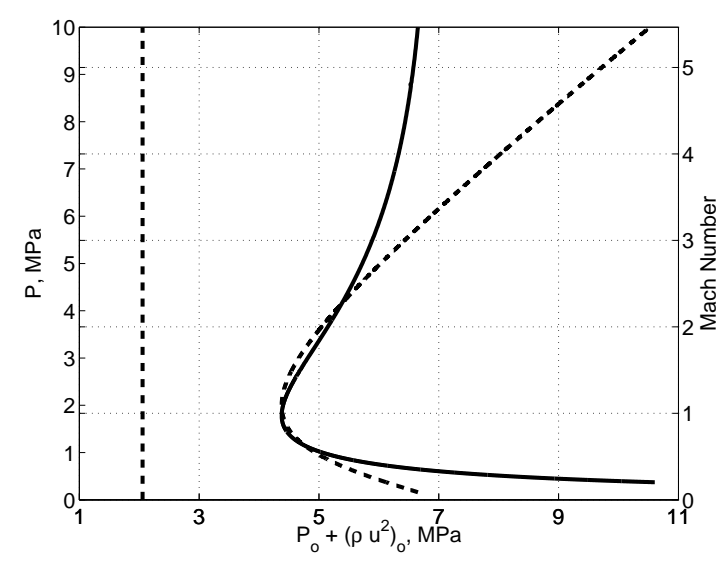

(c) hydrogen-oxygen-nitrogen system, solutions.

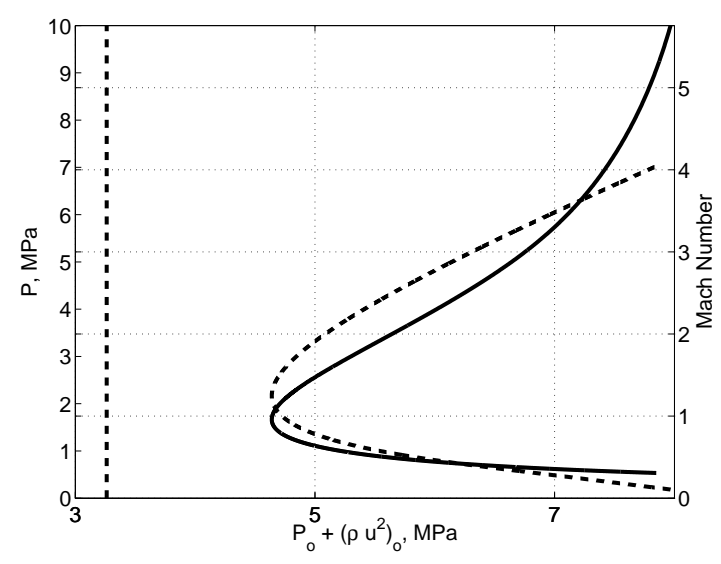

(e) propane system, solutions.

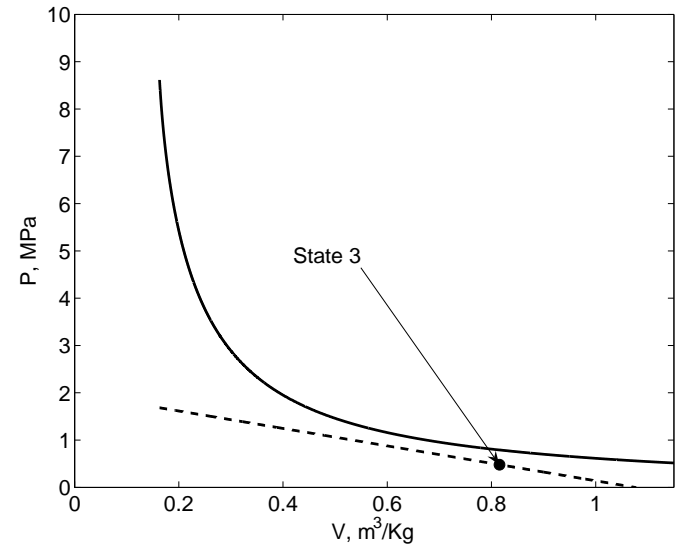

(b) same as (a), $P-V$ plane.

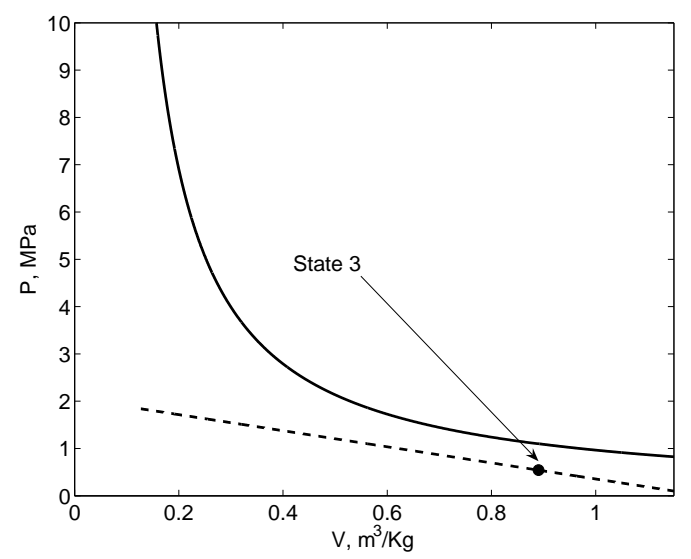

(d) same as (c), $P-V$ plane.

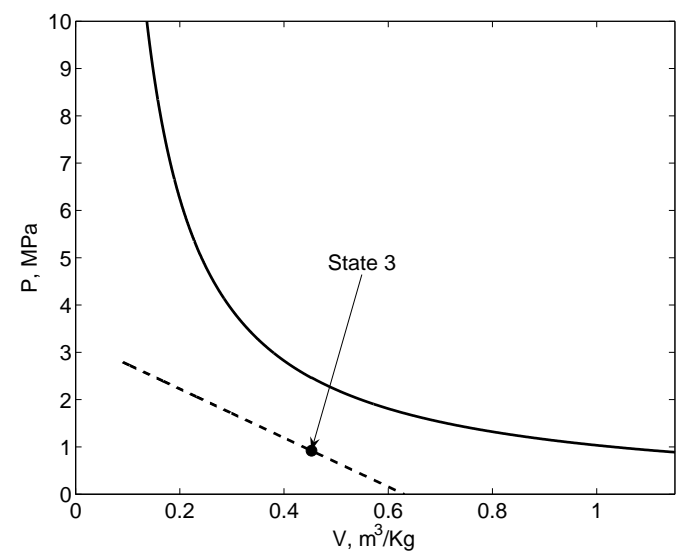

(f) same as (e), $P-V$ plane.

Figure 9: Equilibrium solutions for the three mixtures. In panels (a,c,e), the dashed line shows pressure and the solid line Mach number. The vertical dashed lines are the shock-polar momentum fluxes. In panels $(\mathrm{b}, \mathrm{d}, \mathrm{f})$, the solid line is the products Hugoniot curve and the dashed line is the Rayleigh line.

American Institute of Aeronautics and Astronautics 
are performed is here denoted as coarse. The figure refers to the hydrogen-oxygen-argon system and the comparison was performed by starting from the same interpolated condition at $t=150 \mu \mathrm{s}$, and integrating in time up to $t=250 \mu \mathrm{s}$. The vortical structures shown in the two subfigures have the same shape and

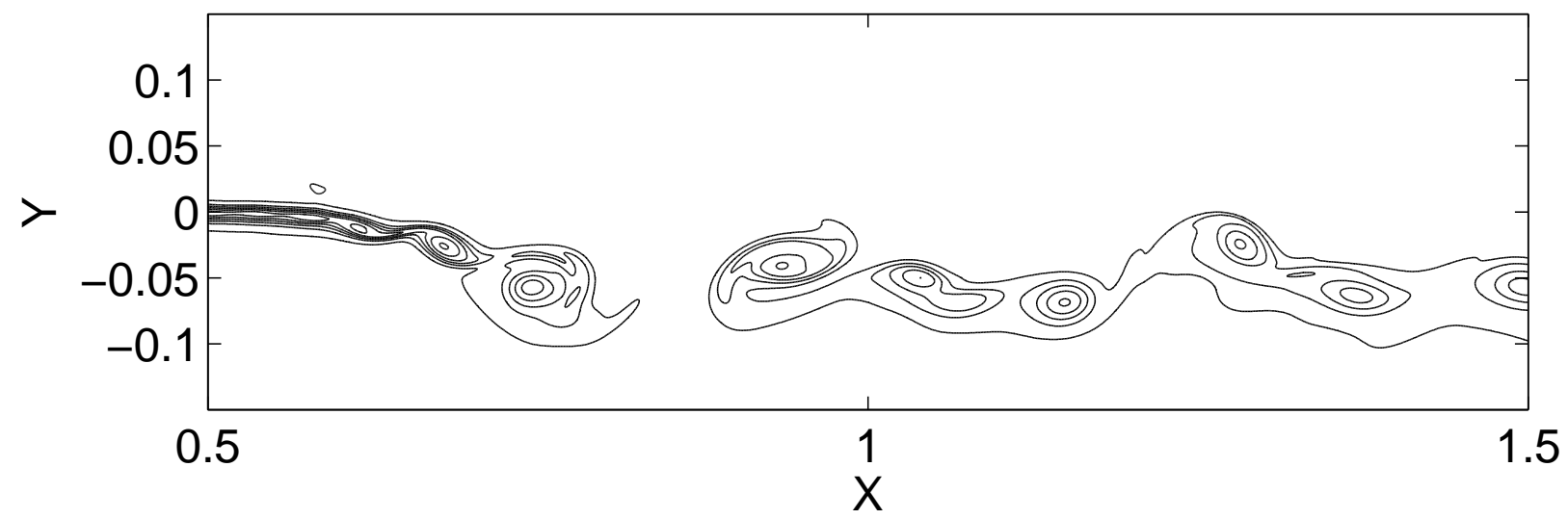

(a) Coarse grid

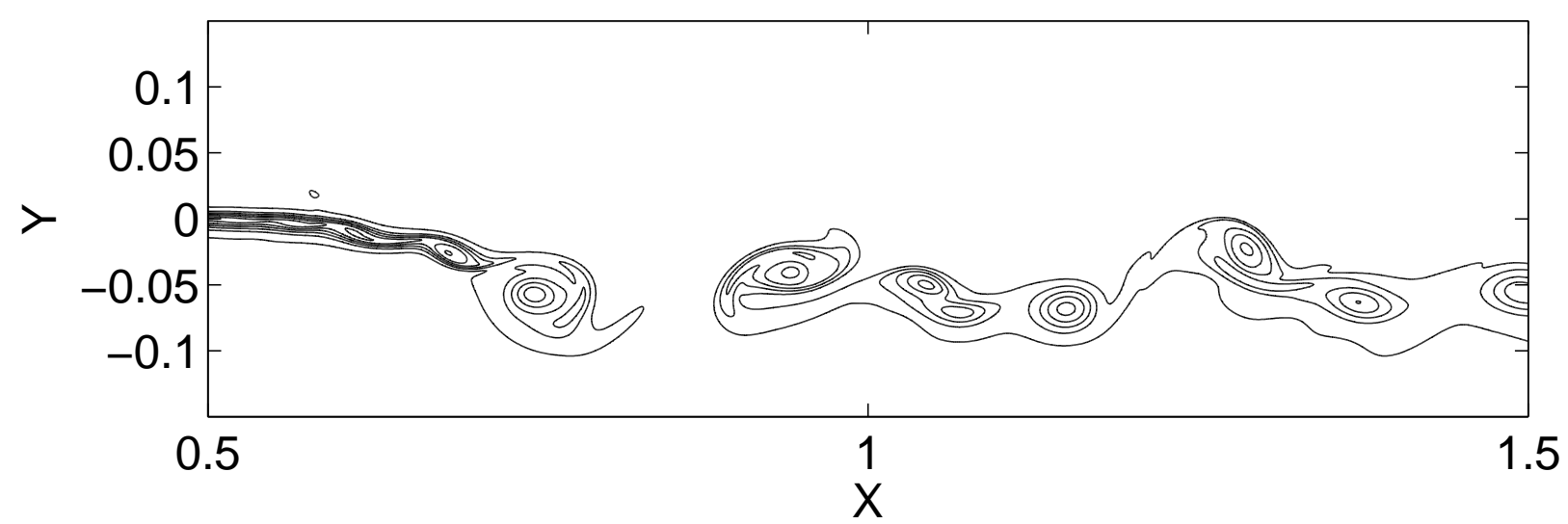

(b) Fine grid

Figure 10: Resolution study. Vorticity, 30 contours in $[-3,0.01] \mu s$. The grids have a total number of mesh points of 827,576 and $1,865,065$.

location, with the highest vorticity contours slightly under-resolved by the coarse grid computations. It is remarked here that a possible source of disagreement between coarse and fine predictions can also be the randomness in the injection boundary velocity.

\section{Numerical experiments}

Numerical results are presented for the three mixtures. In all the 2-dimensional plots the spatial dimensions are expressed in $\mathrm{cm}$. A snap shot of the solution field for the hydrogen-oxygen-argon system at time $30 \mu \mathrm{s}$ is presented in Fig. 11. This time scale corresponds approximately to the detonation cell cycle period for this mixture. The spatial development of the instability match well with experiments. Fig. 11(b) shows that ignition occurs at a distance of approximately $0.5 \mathrm{~cm}$ from the injection. Large scale vortical structures develop beyond the ignition distance, so that the instability does not have a noticeable effect on the thermal 
runaway. On the other hand, the ignition of the cold stream appears to slow down the formation and growth of vorticity structures. Fig. 11(d) shows the Mach number, $M$. The single contour shows that in the cold stream $M$ approaches unity at two locations, but the flow never passes through the sonic line; the hot stream is always subsonic. The outcome that the sonic condition is never reached, in contrast to the one dimensional result, can be attributed to stream tube divergence or unsteadiness or both. The stream tube divergence can be associated to the cross area dilatation,

$$
\delta_{a}=\frac{1}{A} \frac{D A}{D t},
$$

which in 2 dimension is evaluated as

$$
\delta_{a}=\frac{\partial v_{n}}{\partial n}=(\nabla \overrightarrow{\boldsymbol{v}} \cdot \overrightarrow{\boldsymbol{n}}) \cdot \overrightarrow{\boldsymbol{n}}
$$

where $\overrightarrow{\boldsymbol{n}}=(-v, u) /\|\overrightarrow{\boldsymbol{v}}\|$ is the normal to the streamline. Contour values of the area dilatation are shown in Fig. 11(e), for two positive values of the variable. The locations of the two regions of large dilatation correspond to the regions of high temperature gradient, Fig. 11(a), and regions of high $O H$ concentration, Fig. 11(b). Moreover, the values of area dilatation in these regions are comparable to those of the volume dilatation field. Therefore, Fig. 11(e) demonstrates a non-negligible contribution by the stream-tube divergence to the dilatation where chemical activity is pronounced.

The two other systems do not support a regular combustion structure, but localized explosion patterns develop in the cold stream. This result is in agreement with the experimental observations of Austin, ${ }^{7}$ and large scale simulations by Khokhlov et al. ${ }^{36}$ who showed that the explosions are associated with large localized pressure gradients. The explosions are unsteady and two-dimensional in nature, and produce shock waves in the supersonic stream that propagate up to the inflow boundary. The simulations are stopped when the shock reaches the inflow boundary. Pressure and vorticity contours for the hydrogen-oxygennitrogen system are shown in Fig. 12. The same quantities for the propane system are shown in Fig. 13. The thick dashed line represents the locus where the temperature has reached $90 \%$ of the burnt equilibrium value. Therefore, this locus roughly represents the position of the transversal ignition front as it propagates in the fresh mixture due to molecular diffusion. A comparison between Fig. 12(b) and Fig. 13(b) reveals that, for the high activation energy case, the burning front has totally covered the region of high vorticity. The high propagation speed of the ignition front causes entrainment of high temperature gas in the cold stream to be negligible. An antithetical observation is valid for the medium activation energy mixture, where entrainment is important and the ignition front interacts with the fluid-dynamic instability.

Figs. 12(a) and 13(a) also show that the explosion are much weaker in the hydrogen-oxygen-nitrogen system than in the propane system. In the latter case, the pressure shock waves spread across the boundary layer reaching the hot stream, while in the medium activation energy case they are confined to the cold stream and propagate one-dimensionally. Vortical structures are markedly more pronounced in the hydrogen-oxygennitrogen system than in the propane system. This is in agreement with the results of the linear analysis.

Figs. 12 and 13 show that explosions develop near the transversal ignition front. The relation between the transversal ignition front and the Kelvin-Helmholtz instability is analyzed by seeking a statistical description of the entrainment process in the time leading to explosion. Entrainment is associated with large scale mixing as it transports particles across the stream layer. Given the equal premixed composition nature of the streams, the entrainment will be characterized with respect to temperature mixing and transport, as this process has the potential to create localized ignition fronts and favor the formation of localized thermal runaway. The magnitude of the entrainment can be analyzed by looking at the probability density function (PDF) for the time sequences defined by the thermal history at fixed locations. The local probability density function, $\Psi(x, y, T)$ are taken over the time interval $[7,10] \mu s$ and the temperature is non-dimensionalized using the temperature difference across the mixing layer,

$$
\hat{T}=\frac{T-T_{-\infty}}{T_{\infty}-T_{-\infty}} .
$$

The time interval was selected on the basis of the time frame over which ignition occurs for the three mixtures. 


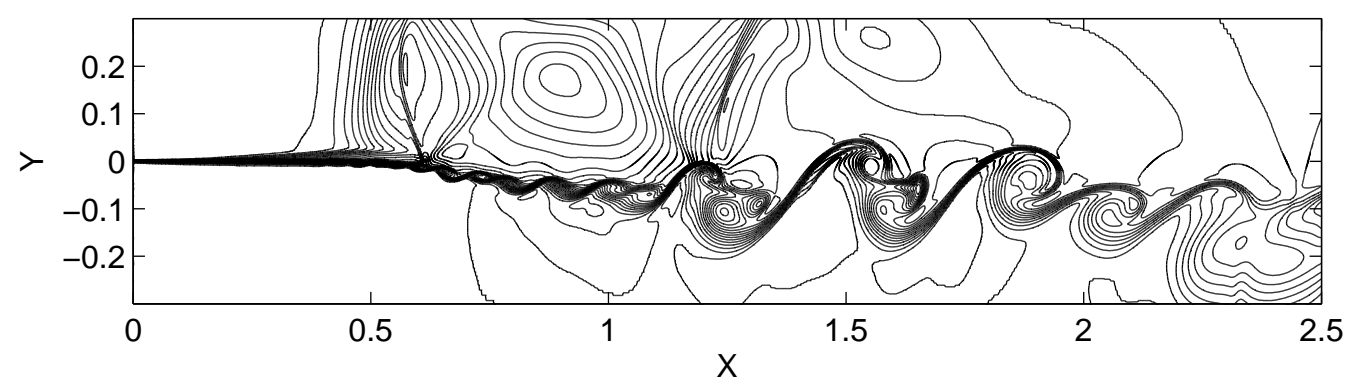

(a) Temperature, 30 contours in $[1700,2800] \mathrm{K}$

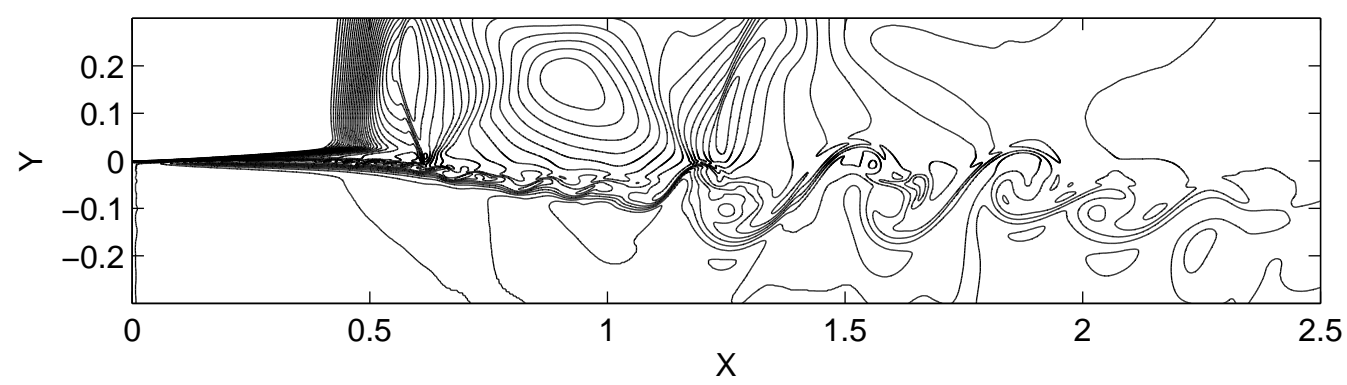

(b) $\mathrm{OH}$ mass fraction, 30 contours in $[0,0.05]$

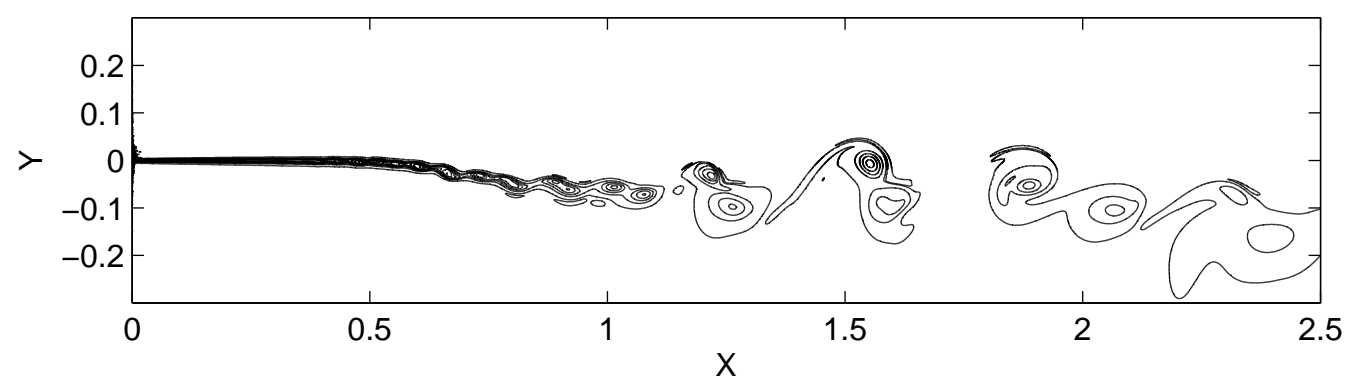

(c) Vorticity, 30 contours in $[-2.5,0.2] \mu s$

Figure 11: Numerical simulation of the hydrogen-oxygen-argon system shear-layer.

24 of 33

American Institute of Aeronautics and Astronautics 


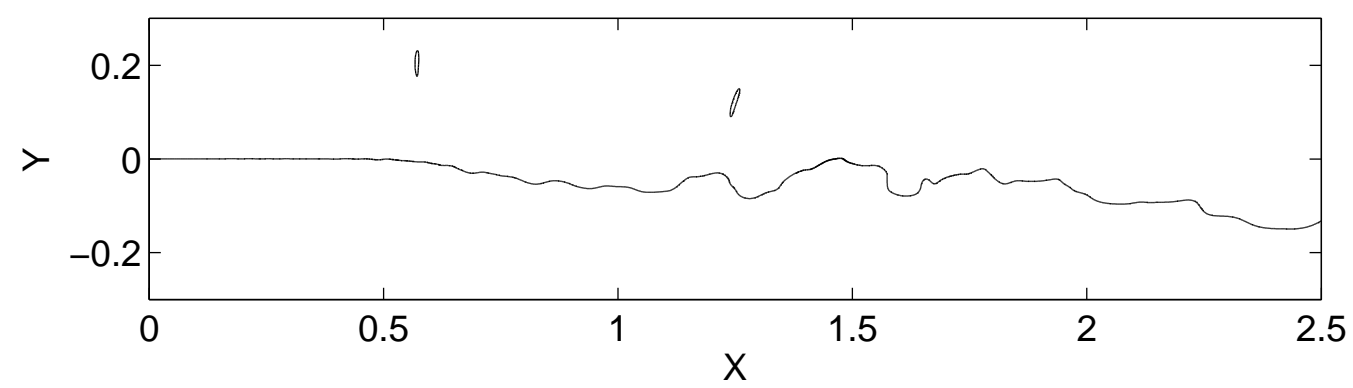

(d) Mach number, one contour drawn for Mach $=1.03$

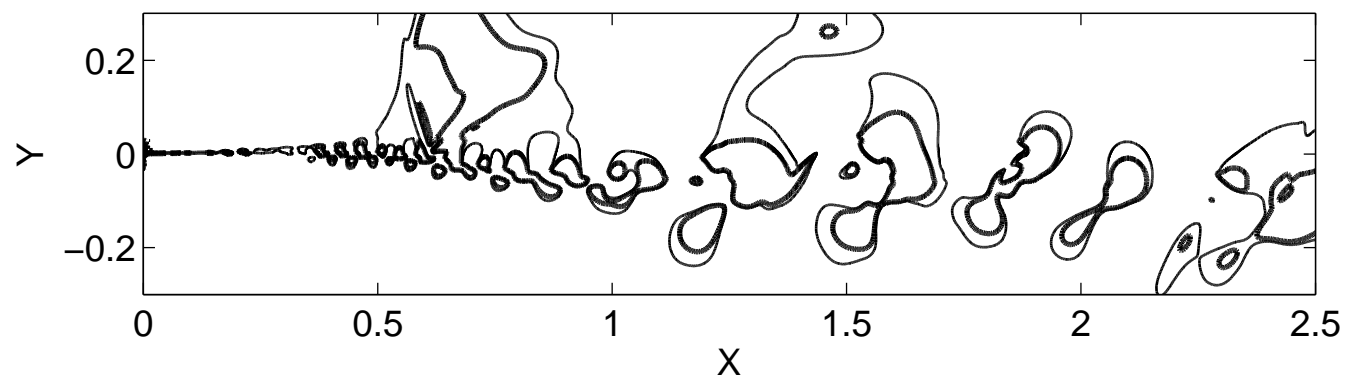

(e) $\frac{\partial v_{n}}{\partial n}$, two contours: $0.05 \mu s$ thin line, and $0.1 \mu s$ thick line

Figure 11: Numerical simulation of the hydrogen-oxygen-argon system shear-layer.

In this context it is useful to provide a statistical description of entrainment by considering $80 \%$ confidence intervals, defined as the minimum interval of values of a random variable that contains $80 \%$ of the data. In this analysis each location in the flowfield is considered separately as a random variable. More specifically the following quantity is considered,

$$
\Delta T_{80}=\min \left\{\Delta T \mid \int_{\hat{T}}^{\hat{T}+\Delta T} \Psi(x, y, T)>0.8\right\} .
$$

The confidence interval is plotted versus the crosswise coordinate in Fig. 14 for two axial locations and for the three mixtures considered.

Fig. 14 shows that the hot fluid entrainment is almost absent in the propane system. The spikes in $\Delta T_{80}$ seen in Figs. 14(b) and 14(c) are due to fluctuation of the transversal ignition front, the one that separates reacted from unreacted gas. The large spike in the propane system is caused by the steepness of its ignition front, a consequence of its high effective activation energy. The levels of entrainment for the small and medium activation energy mixtures are comparable. The hydrogen-oxygen-argon system has a larger maximum value of $\Delta T_{80}$ than hydrogen-oxygen-nitrogen system. The difference between maximum $\Delta T_{80}$ values for the medium and low activation energy cases increases with the axial distance in favor of the low $\theta_{\text {eff }}$ case. This phenomenon is linked to the difference in propagation speed of transversal ignition front as it moves into the fresh mixture. The location of the ignition front controls the effective temperature difference available for large scale mixing across the shear-layer. The link between ignition front propagation speed and mixture activation energy is considered valid based upon the numerical simulations.

These concepts can be substantiated by looking at the probability density functions for the scaled temperature at $x-x_{0}=0.5 \mathrm{~cm}$, and at the maximum $\Delta T_{80}$ crosswise coordinate location. The PDFs for the three mixtures are shown in Fig. 15. The figure represents a situation typical in large scale mixing, where the PDF has peaks at the two extrema of the temperature interval: the peaks correspond to the temperature 

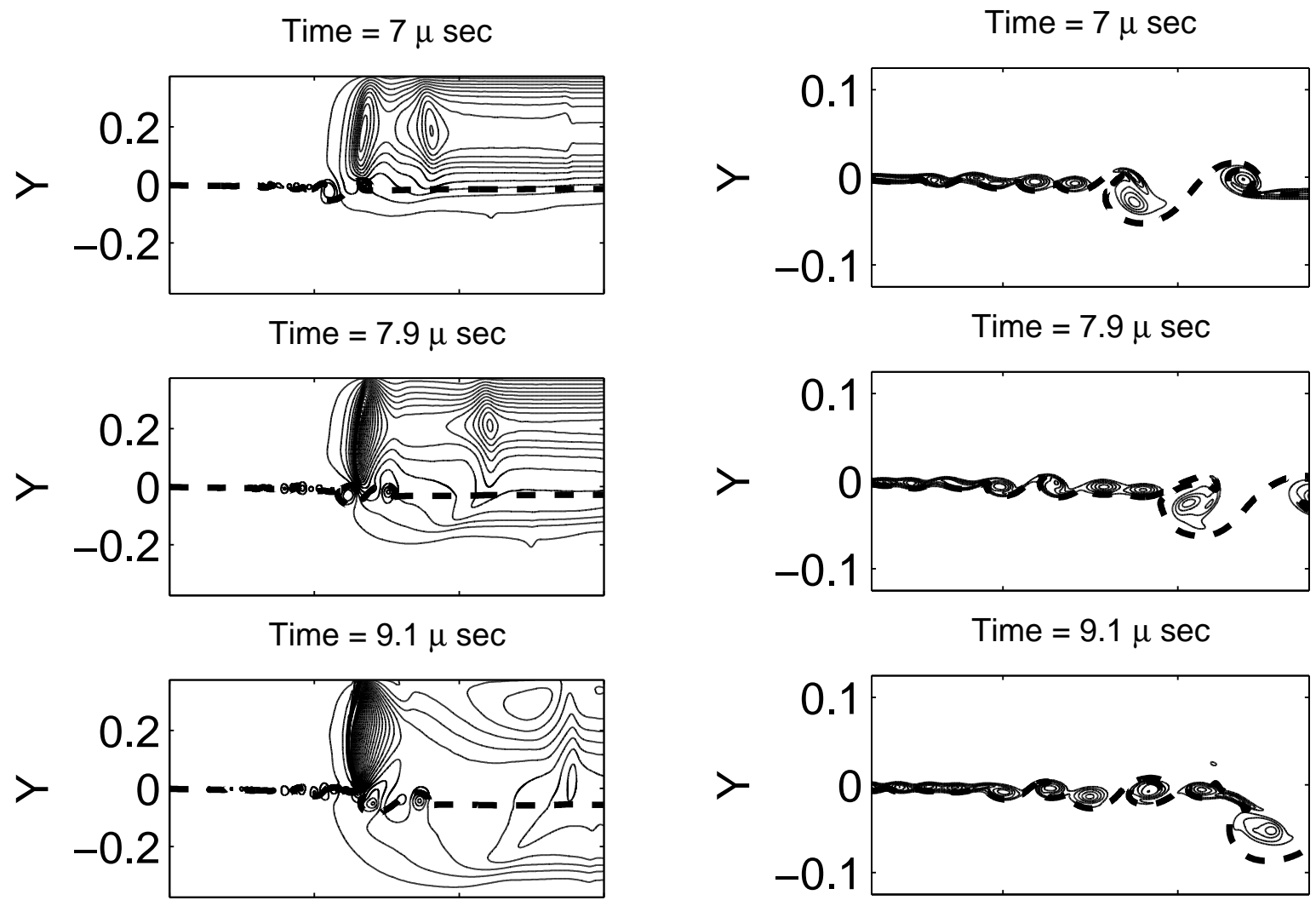

Time $=10.1 \mu \mathrm{sec}$

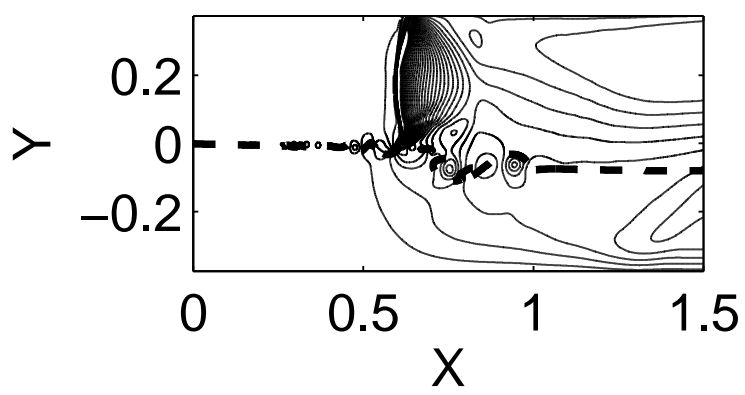

(a) Pressure, 30 contours in $[0.5,1.5] \mathrm{MPa}$

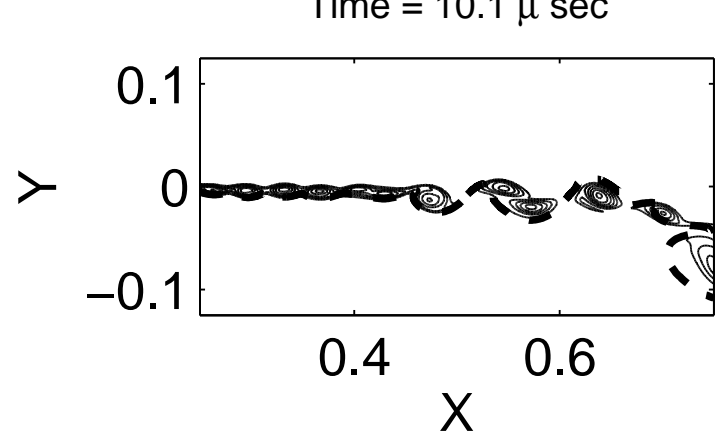

(b) Vorticity, 30 contours in $[-4,0.2] \mu s$

Figure 12: Numerical simulation of the hydrogen-oxygen-nitrogen system shear-layer. The thick dashed line in both panels represents the locus where the temperature has reached $90 \%$ of its equilibrium value. 

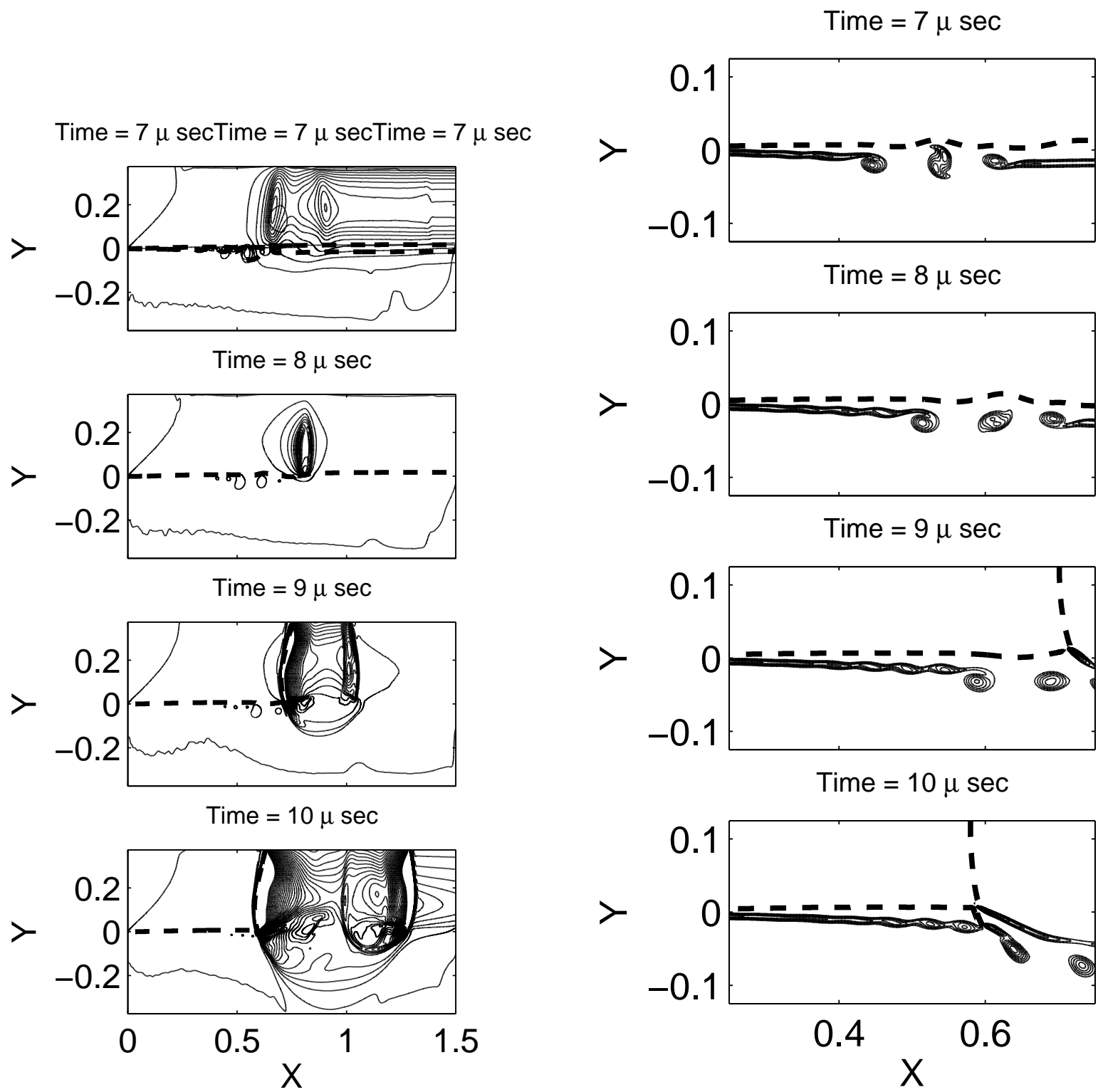

(a) Pressure, 30 contours in $[0.85,3] \mathrm{MPa}$

(b) Vorticity, 30 contours in $[-2,-0.2] \mu \mathrm{sec}$

Figure 13: Numerical simulation of the propane system shear-layer. The thick dashed line in both panels represents the locus where the temperature has reached $90 \%$ of its equilibrium value. 


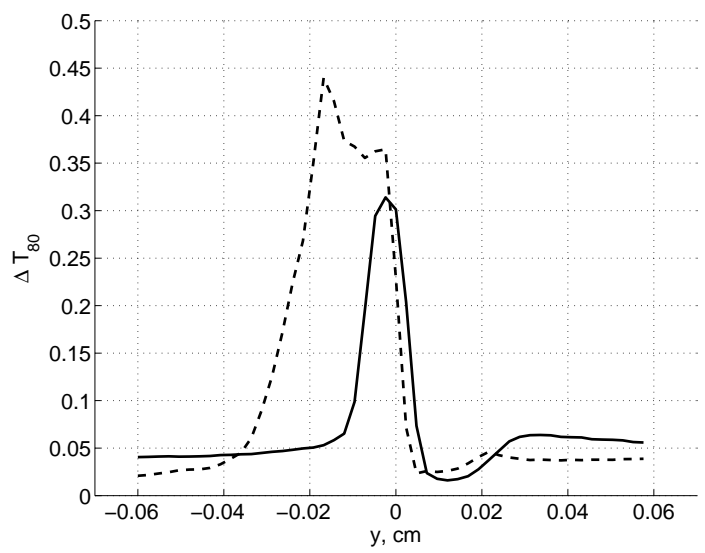

(a) hydrogen-oxygen-argon system

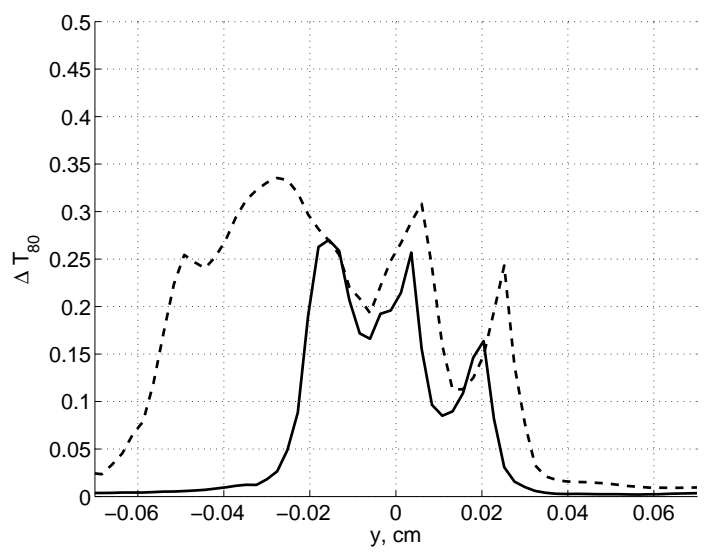

(b) hydrogen-oxygen-nitrogen system

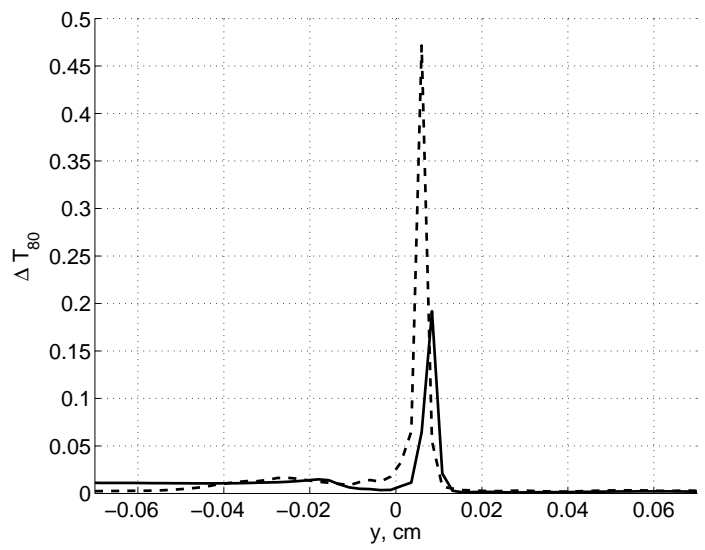

(c) propane system

Figure 14: $80 \%$ confidence intervals for the scaled temperature. The solid line is taken at $x-x_{0}=0.5 \mathrm{~cm}$ while the dashed line at $x-x_{0}=0.6 \mathrm{~cm}$.

28 of 33

American Institute of Aeronautics and Astronautics 


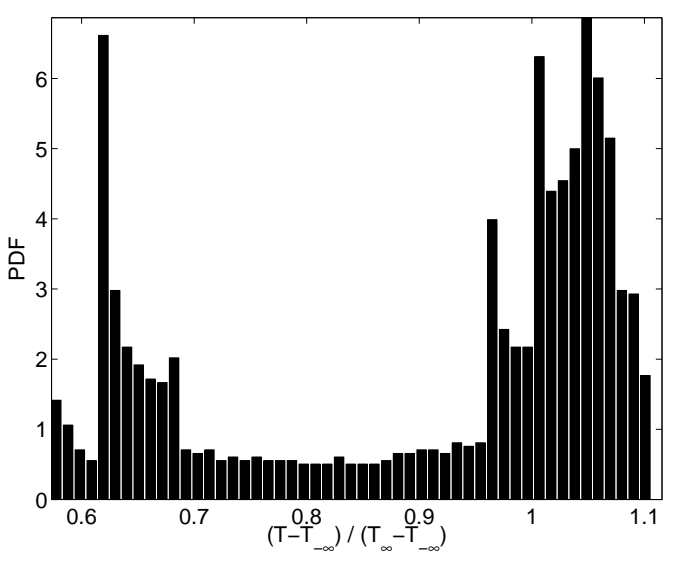

(a) hydrogen-oxygen-argon system

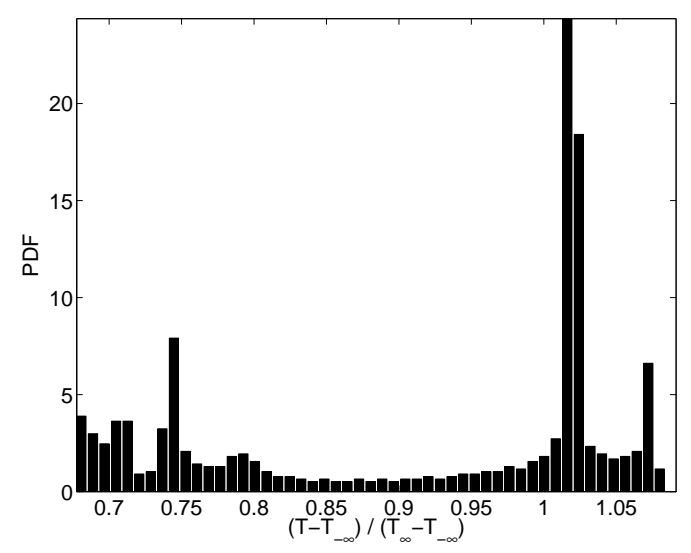

(b) hydrogen-oxygen-nitrogen system

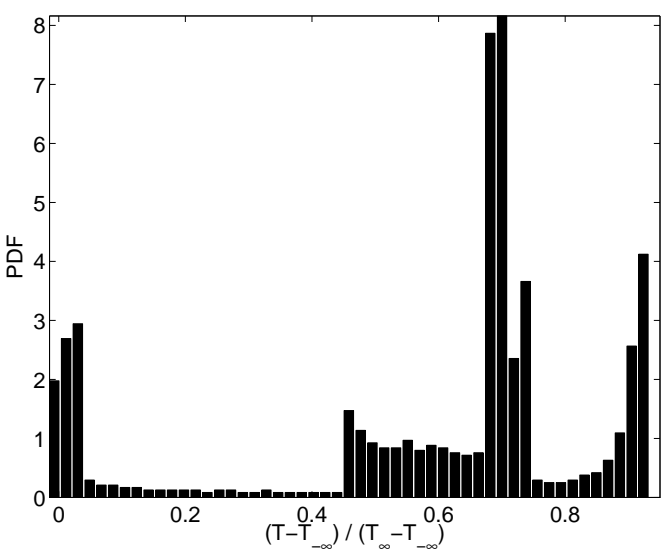

(c) propane system

Figure 15: PDF of the scaled temperature taken at $x-x_{0}=0.5 \mathrm{~cm}$ and at a $y$ location which correspond to the maximum value of $\Delta T_{80}$. Note that the slight region of negative values for panel (c) is due to the fact that the gas temperature drops lower than the cold stream value for the hydrocarbon mixture. 29 of 33

American Institute of Aeronautics and Astronautics 
of the two mixed fluids. It must be recognized in Fig. 15(c) that entrainment mixing is responsible for temperature observations in the interval [0.5,1], while the lower part of the PDF is due to fluctuation of the transversal injection front. Note the negative values of $\Delta T_{80}$ in Fig. 15(c). They can be explained by noting that the gas temperature falls below the cold stream value for the hydrocarbon mixture at the edges of the mixing region, a phenomenon associated with the early stages of reaction in the propane system mixture.

\section{Conclusions}

The analysis presented in this paper was motivated by the experimental observations of localized explosions, and local regions of intense luminescence in the region of triple point shear-layers in propagating gaseous detonation waves. These features were only observed in mixtures with higher effective activation energy. The correlation between mixture activation energy and the appearance of localized "hot spots" has been examined by performing linear stability analysis of the shear-layers and 2D Navier Stokes calculations with detailed chemical kinetics and transport. The inflow conditions for the simulations are based on shock polar calculations for conditions on either side of the triple point contact surface in three mixtures characterized by a ratio in effective activation energy $\theta_{\text {eff,max }} / \theta_{\text {eff,min }} \approx 2.35$. These mixtures are chosen to correspond to the experimental conditions.

The linear analysis has shown that the large effective activation energy mixtures have lower spatial growth rates than low activation counterparts, and that the mean profile is mostly responsible for the growth rate attenuation. It is concluded that the decoupling between velocity gradient and thermal gradient is the underlying mechanism. This observation establishes a correlation between activation energy and stability, but one that excludes the possibility that the localized ignition fronts are associated with an enhanced shear layer instability for the high activation energy systems.

A set of two dimensional simulations predict localized ignition fronts for the high activation energy case. These localized explosions are unsteady and two-dimensional, originate close to the transversal ignition front and rapidly propagate through the mixture. The advection of the localized ignition fronts in the fresh mixture is through shock waves. The "hot spots" are characterized by large pressure peaks, the temperature is well above that of the constant pressure equilibrium, and a high concentration of $O H$ mass fraction is observed. All these facts are consistent with the experiments.

The interaction between mixture ignition and the development of the instability has been analyzed by considering the entrainment of high temperature gas in the mixing region. The simulations have shown that the level of entrainment diminishes with an increase in effective activation energy, and it is almost absent in the highest activation energy mixture. This outcome is agreement with the linear analysis but also shows that, for the high activation energy mixtures, the high speed of propagation of the transversal ignition front causes the high temperature/reacted gas region to completely consume the high vorticity region. The realization that the propagation speed of the transversal front is diffusion controlled points to the importance of diffusion effects in detonation systems.

In conclusion, shear layer instability appears to play no role in the formation of localized explosions. Molecular diffusion heating is responsible for the fact that high reactivity pockets originate close to the transversal ignition front. The explosions are instead associated with the impossibility of reaching a 1D equilibrium state in the cold stream in the shock frame. Such a lack of equilibrium is at the basis of the localized and multidimensional nature of the ignition for the high activation energy case. The low activation energy system supports a regular solution devoid of localized ignition fronts, even though equilibrium 1D states do not exist in the natural shock frame. The stream-tube cross-section area expansion is the key factor in the stabilization of the flow. The reason why the higher activation energy mixtures are not stabilized is probably related to the much faster volume build-up at ignition. 


\section{Acknowledgments}

LM and TLJ were supported by the U.S. Department of Energy through the University of California under subcontract number B341494. This work was supported in part by funding from the ONR with Dr. Gabriel Roy as technical monitor. 


\section{References}

${ }^{1}$ White, D., "Turbulent structure of gaseous detonation," Phys. Fluids, Vol. 4, 1961, pp. 465-480.

${ }^{2}$ Voitsekhovskii, B. V., Mitrofanov, V. V., and Topchian, M. E., "Struktura fronta detonastii i gaza," Akad. Nauk., SSSR, Novosibirsk, 1963, Translation: The structure of a detonation front in gases Rep. FTD-MT-64-527, Foreign Technology Division, Wright-Patterson A.F.B., Ohio,(1966).

${ }^{3}$ Edwards, D. H., Hooper, G., and Meddins, R. J., "Instabilities in the reaction zones of detonation waves," Astronautica Acta, Vol. 17, No. 4-5, 1972, pp. 475-485. 315 .

${ }^{4}$ Strehlow, R. and Crooker, A., "The structure of marginal detonation waves," Acta Astronautica, Vol. 1, 1974, pp. 303-

${ }^{5}$ Takai, R., Yoneda, K., and Hikita, T., "Study of detonation wave structure," 15th Symposium (International) on Combustion, The Combustion Institute, Pittsburgh, PA, 1974, pp. 69-78.

${ }^{6}$ Pintgen, F., Eckett, C. A., Austin, J. M., and Shepherd, J. E., "Direct Observations of Reaction Zone Structure in Propagating Detonations," Combust. Flame, Vol. 133, No. 3, 2003, pp. 211-229.

${ }^{7}$ Austin, J. M., The Role of Instability in Gaseous Detonation, Ph.D. thesis, California Institute of Technology, Pasadena, California, May 2003.

${ }^{8}$ Austin, J., Pintgen, F., and Shepherd, J., "Lead shock oscillation and decoupling in propagating detonations," AIAA, , No. 05-1170, 2005.

${ }^{9}$ Austin, J., Pintgen, F., and Shepherd, J., "Reaction zones in highly unstable detonations," Proc. Combustion Insitute, Vol. 30, 2005, pp. 1849-1857.

${ }^{10}$ Gamezo, V. N., Desbordes, D., and Oran, E. S., "Two-dimensional reactive flow dynamics in cellular detonation waves," Shock Waves, Vol. 9, 1999, pp. 11-17.

${ }^{11}$ Urtiew, P. A., "Reflections of wave intersections in marginal detonations." Astronautica Acta, Vol. 15, 1970, pp. 335-343.

${ }^{12}$ Strehlow, R., "The natue of transverse waves in detonations," Astronautica Acta, Vol. 14, 1969, pp. 539-548.

${ }^{13}$ Oppenheim, A., Smolen, J., and Zajac, L., "Vector polar method for the analysis of wave intersections," Combust. Flame, Vol. 12, 1968, pp. 63-76.

${ }^{14}$ Gamezo, V. N., Desbordes, D., and Oran, E. S., "Formation and evolution of two-dimensional cellular detonations," Combust. Flame, Vol. 116, 1999, pp. 154-165.

${ }^{15}$ S.Singh, Leiberman, D., and J.E.Shepherd, "Combustion behind shock waves," Western States Section, The Combustion Institute. Paper 03F-29, 2003.

${ }^{16}$ Arienti, M. and Shepherd, J., "The role of diffusion at shear layers in irregular detonations," Joint Meeting of the U.S. Sections of the Combustion Institute, Philadelphia, PA, 2005.

${ }^{17}$ Subbotin, V., "Two kinds of transverse wave structures in multi-front detonation," Fizika Goreniya $i$ Vzryva, Vol. 11, No. 1,1975 , pp. $96-102$.

${ }^{18}$ Lundstrom, E. A. and Oppenheim, A. K., "On the influence of non-steadiness on the thickness of the detonation wave," Proc. Roy. Soc. A, Vol. 310, 1969, pp. 463-478.

${ }^{19}$ Jackson, T. and Grosch, C., "Absolute/Convective instability and the convective mach number in a compressible shea layer," Phys. Fluids, Vol. 2, No. 6, 1990, pp. 949-954.

${ }^{20} \mathrm{Konnov}, \mathrm{A}$., "Development and validation of a detailed reaction mechanism for the combustion of small hydrocarbons," 28th Symp. (Intl) on Combustion, The Combustion Institute, 2000, p. 317.

${ }^{21}$ Schultz, E. and Shepherd, J. E., "Validation of detailed reaction mechanisms for detonation simulation," Tech. Rep. FM99-5, Graduate Aeronautical Laboratories: California Institute of Technology, 2000.

${ }^{22}$ Smith, G. P., Golden, D. M., Frenklach, M., Moriarty, N. W., Eiteneer, B., Goldenberg, M., Bowman, C. T., Hanson, R. K., Song, S., Gardiner, W. C., Jr., Lissianski, V. V., , and Qin, Z., "GRI-Mech 3.0," http://www.me.berkeley.edu/gri_mech/.

${ }^{23}$ Criminale, W., Jackson, T., and Joslin, R., Theory and computation of hydrodynamic stability, Cambridge Monographs on Mechanics, Cambridge University Press, 2003.

${ }^{24}$ Shin, D. and Ferziger, J., "Linear Stability of the Compressible Reacting Mixing Layer," AIAA Journal, Vol. 31, 1993, pp. $677-685$.

${ }^{25}$ Dixon-Lewis, G., "Flame structure and flame reaction kinetics. II. Transport phenomena in multicomponent systems," Proc. R. Soc. Lond. A, Vol. 307, No. 1488, 1968, pp. 111-135.

${ }^{26}$ Kee, R. J., Dixon-Lewis, G., Warnatz, J., Coltrin, M. E., and Miller, J., "A FORTRAN Computer Code Package for the Evaluation of Gas-Phase Multicomponent Transport Properties," Report SAND86-8246, Sandia National Laboratories, 1986.

${ }^{27}$ Kee, R. J., Rupley, F. M., Meeks, E., and Miller, J. A., "CHEMKIN-III: A FORTRAN chemical kinetics package for the analysis of gasphase chemical and plasma kinetic," Report SAND96-8216, Sandia National Laboratories, 1996.

${ }^{28}$ Lock, R., "The velocity distribution on the laminar boundary layer between parallel streams," Quart. J. Mech. Appl. Math., Vol. 4, 1951, pp. 42-.

${ }^{29}$ Brankin, R., Gladwell, I., and Shampine, L., "rksuite," http://www.netlib.org.

${ }^{30}$ Jackson, T. and Grosch, C., "Inviscid spatial stability of a compressible mixing layer. Part 3. Effect of thermodynamics." J. Fluid Mech., Vol. 224, 1991, pp. 159-175. 
${ }^{31}$ Hung, P. and Shepherd, J., "Reduction of detailed chemical reaction networks for detonation simulations," 12th International Detonation Symposium, 2002.

${ }^{32}$ Day, M. J., Reynolds, W. C., and Mansour, N. N., "The structure of the compressible reacting mixing layer: Insights from linear stability analysis," Phys. Fluids, Vol. 10, No. 4, 1998, pp. 993-1007.

${ }^{33}$ Kennedy, C. and Carpenter, M., "Comparison of Several Numerical Methods for Simulation of Compressible Shear Layers," Technical Paper 3484, NASA, December 1997.

${ }^{34}$ Kennedy, C. and Carpenter, M., "Several new numerical methods for compressible shear layer simulations," Applied Numerical Mathematics, Vol. 14, 1994, pp. 397-433.

${ }^{35}$ Poinsot, T. and Lele, S., "Boundary Conditions for Direct Simulations of Compressible Viscous Flows," J. Comput. Phys., Vol. 101, 1992, pp. 104-129.

${ }^{36}$ Khokhlov, A., Austin, J., Pintgen, F., and Shepherd, J., "Numerical study of the detonation wave in ethylene-oxygen mixtures," No. AIAA-2004-0792, AIAA, 2004. 